\title{
Design considerations for mesoporous silica nanoparticulate systems in facilitating biomedical applications
}

\begin{abstract}
Mesoporous silica nanoparticles (MSNs) have advanced to the forefront of multifunctional nanoparticulate systems in nanomedicine, owing to this highly flexible materials platform enabling a multitude of design options, often in a modular fashion. Drug delivery ability, detectability via different imaging modalities, and stimuliresponsiveness are often combined into one particle system. Very sophisticated and versatile designs along with impressive demonstrations of applicability have been reported to date, but a common ground when it comes to some critical considerations valid for any nanoparticle intended for biomedical purposes is lacking to some degree. In this study, we attempt to take a glance at some of the most crucial aspects of biomedical nanoparticulate design and relate how they apply specifically to MSNs. These considerations include fluorophore labeling and leaching with respect to immobilization to MSNs, the surrounding conditions, carrier biodegradability, and surface coating. Surface modification strategies and surface charge tuning are further considered in conjunction to the relative amount of cellular uptake and serum protein adsorption. Cellular internalization routes and biological techniques used to evaluate especially in vitro biobehavior are discussed. Our attempt is hereby to draw attention to some of the most frequently occurring issues to be considered in the design of MSN systems for biomedical applications.
\end{abstract}

Keywords: fluorophore labeling; fluorescence properties; photostability; surface coating; surface charge; cellular uptake; cellular internalization routes; serum protein adsorption; PEI cytotoxicity

Diti Desai: Center for Functional Materials, Laboratory for Physical Chemistry, Åbo Akademi University, Finland

Diti Desai: Pharmacy Department, Faculty of Tech. \& Eng., The M.S. University of Baroda, India

Didem Şen Karaman: Center for Functional Materials, Laboratory for Physical Chemistry, Åbo Akademi University, Finland

Neeraj Prabhakar: Center for Functional Materials, Laboratory for Physical Chemistry, Åbo Akademi University, Finland
Neeraj Prabhakar: Laboratory of Biophysics, Institute of Biomedicine and Medicity Research Laboratories, University of Turku, Finland

Sina Tadayon, Alain Duchanoy: Center for Functional Materials, Laboratory for Physical Chemistry, Åbo Akademi University, Finland Diana M. Toivola: Department of Biosciences, Cell biology, Åbo Akademi University, Finland

Sadhana Rajput: Pharmacy Department, Faculty of Tech. \& Eng., The M.S. University of Baroda, India

Tuomas Näreoja: Laboratory of Biophysics, Institute of Biomedicine and Medicity Research Laboratories, University of Turku, Finland *Corresponding Author: Jessica M. Rosenholm: Center for Functional Materials, Laboratory for Physical Chemistry, Åbo Akademi University, Finland, E-mail: jerosenh@abo.fi

\section{Introduction}

In recent years, mesoporous silica nanoparticles (MSNs) have received increasing attention in the field of nanomedicine, largely due to their wide design flexibility and extensive applicability [1-11]. Among other advantages, their surfaces can be easily and selectively functionalized, they possess uniform mesopores for efficient guest molecule loading and release, with sufficient safety and significant biocompatibility. In the current race for more complex designs capable of concurrently performing a multitude of tasks, MSNs are especially well-suited for incorporating the essential capabilities of a theranostic platform into a single particle, with separate domains for (1) the contrast agent/optical label that enables traceable imaging of target, (2) the drug payload for therapeutic intervention and (3) the biomolecular ligand for targeted delivery. For any nanomedical device, physicochemical properties such as surface characteristics of the particles play a key role that provides dispersion stability in the physiological environment and give access to develop further selective functionalization regimes, which allows enhancement of the level of sophistication as well as expands the applicability of the nanoparticles. Thorough investigation of the physicochemical characteristics of 


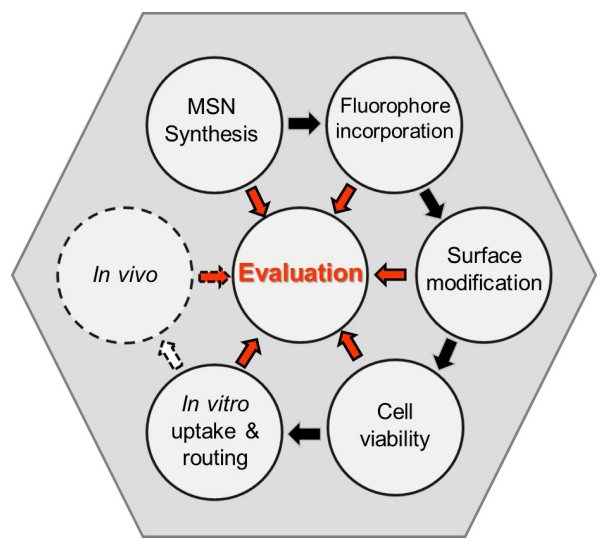

Chart 1. Schematic representation of the design- and evaluation aspects being discussed in this study.

nanoparticles and their correlation to in vitro biobehavior is, therefore, inevitable in aiming for a nanomedical platform with controllable properties. Consequently, a traceable particle with modifiable surface properties lays the foundation for any nanoparticulate system to be utilized in bio/nanomedical device design.

In light of the above, we thus attempted to investigate the effect of a variety of surface modifications on the in vitro behaviour of MSNs in terms of detectability by fluorescence-based techniques, cellular internalization and uptake routes, as well as serum protein adsorption and cytotoxicity according to sequential evaluations as represented in Chart 1 . Since silica is an optically transparent material, MSNs need to be labeled with imaging agents to be traceable in the biological/physiological environment. For this reason, fluorescent tags are most often employed, which makes MSNs possible to study using the most common biological techniques. However, it is well-known that organic fluorophores suffer from certain drawbacks that may limit or at least complicate the quantitative data that can be acquired based on fluorescencebased analyses. Upon immobilization to MSNs, additional parameters come into play, which needs to be considered if aiming toward such quantifications. In order to shed some light on the parameters at play, here we adopted two of the most common fluorescent tags: fluorescein (a pH-dependent fluorochrome) and rhodamine (regarded as pH-independent) and assessed how parameters such as surrounding (biologically relevant) media, particle concentration and especially, surface functionalization may influence the fluorescence properties of fluorescently tagged MSNs. Since surface functionalization is an integral part of MSN design for biomedical applicability, particular emphasis was put on different surface functionalization aspects throughout the study. Thus, to span a broad particle surface charge range, MSNs with positive, neutral, and negative surface charges were included for the implications of the observations related to biobehavior to be as widely applicable as possible. As amine groups are the mostly exploited in bioconjugation reactions, aminosilane co-condensed MSNs are the widely employed as biomedical MSN platforms. For this reason, both an amino-co-condensed as well as pristine silica MSNs were considered. The further surface modification of MSNs was carried out either by direct derivatization of the MSN surface via the primary amine groups introduced by cocondensation, or mediated by a poly(ethylene imine), PEI, layer. PEI was introduced via two different modification strategies, either via electrostatic adsorption (for pristine MSNs) or surface hyperbranching polymerization (for cocondensed MSNs). Subsequently, the surface amines were modified with either poly(ethylene glycol), PEG ("PEGylation") or succinic anhydride ("succinylation"), the latter yielding terminal carboxylic acid groups, the ratio of which can be utilized to fine-tune the surface charge of the particles.

Here, PEG was chosen as it is the most commonly applied surface coating when aiming at 'stealth' (longcirculating) nanoparticles, whereas coatings with zwitterionic properties have also been proposed as an alternative to PEG in reducing plasma protein adsorption [12]. Derivatizing the hyperbranched PEI-layer with terminal carboxylic acid groups results in such a zwitterionic coating containing tertiary, secondary, and possibly residual primary amines as basic/positively charged groups and carboxylic acid groups as acidic/negatively charged groups. To investigate whether this zwitterionic coating could reduce unspecific protein binding, the effect of surface functionalization on the extent of serum protein adsorption was also deduced. Further, the uptake efficiency as a function of surface modification/charge was studied in two different cancer cell lines of different origin, to investigate whether the endocytosis of MSN could be manipulated by surface functionalization and to which extent. Also different routes of uptake, which is highly decisive for the fate of the particle after internalization, have been proposed in the literature to be dependent on especially surface charge [13], which is why this aspect was also considered. Given the versatility of PEI as a platform for further functionalization, special consideration was given to the cytotoxic effect of this surface coating, as high-molecular weight PEI by itself is well-known to be cytotoxic. In summary, we demonstrate how fluorescence variability and stability, cellular uptake and related biobehaviour of MSNs can be manipulated by different surface functionalization 
strategies to conduce towards a rational design platform for MSNs in bio/nanomedicine.

\section{Experimental Procedures}

\subsection{Synthesis and surface modification of fluorophore-incorporated MSNs}

\subsubsection{Preparation of $\mathrm{MSN}_{1}$ series}

Mesoporous silica nanoparticle $\left(\mathrm{MSN}_{1}\right)$ synthesis was carried out by using a similar protocol as in our previous study [14]. Briefly, cetyltrimethylammonium bromide (CTAB) as structure-directing agent (SDA) was dissolved in the ethanolic basic aqueous reaction solution. In this reaction, tetraethylorthosilicate (TEOS) was used as silica source. The reaction was kept stirring for overnight. The molar composition of the synthesis solution was 1TEOS:0.122 CTAB:0.31NaOH:72.3EtOH :946 $\mathrm{H}_{2} \mathrm{O}$ - from [14]. Fluorescent labeling of particles were carried out by using two different amine reactive fluorophores, fluorescein isothiocyanate (FITC) and tetramethylrhodamine isothiocyanate (TRITC). In order to utilize the advantage of easy incorporation of the these fluorophores in the silica matrix, pre-reaction of the fluorophores with aminopropyl triethoxysilane (APTES) was carried out in $2 \mathrm{ml}$ of ethanol with a molar ratio of 1:3 and stirred for 3 hours under inert atmosphere. The pre-reaction solution was added to the reaction solution right before adding the TEOS to the synthesis. The molar ratio between APTES and TEOS was kept as 1:100. After overnight reaction of the MSNs, the SDA was removed by sonication extraction three times in ethanolic $\mathrm{NH}_{4} \mathrm{NO}_{3}$ solution. These FITC and TRITC incorporated particles were denoted F-MSN ${ }_{1}$ and T-MSN $\mathrm{N}_{1}$, respectively. In these two differently labeled particles all the reaction conditions were kept the same, except for the reaction temperature. The F-MSN 1 particles reaction was carried out by elevating the reaction temperature to $80^{\circ} \mathrm{C}$ and maintaining this temperature overnight, whereas the T-MSN 1 particles were carried out at $30^{\circ} \mathrm{C}$ in order to provide difference in pore ordering of the particles by elevating the reaction temperature [15] when using this as a parameter later in the study.

The modification of MSNs were provided by electrostatic adsorption of branched $25 \mathrm{k}$ poly(ethylene imine) (PEI) on the particle surfaces. The functionalization of MSNs was carried out by overnight adsorption in 4(2-hydroxyethyl)-1-piperazineethanesulfonic acid (HEPES) buffer at neutral $\mathrm{pH}$ and also in ethanol in order to investigate the alteration in porous order of the particles during the surface modification. After surface modification, the particles were collected by centrifugation and washed to remove excess $\mathrm{PEI}$. These particles were denoted $\mathrm{PEI}_{a d s}-\mathrm{F}$ $\mathrm{MSN}_{1}$ and $\mathrm{PEI}_{a d s}-\mathrm{T}-\mathrm{MSN}_{1}$.

\subsubsection{Preparation of $\mathrm{MSN}_{2}$ series}

Preparation of $\mathrm{MSN}_{2}$ series $\mathrm{MSN}_{2} \mathrm{~S}$ were synthesized according to our previously published protocols [16] where methanol was used as co-solvent and cetyltrimethylammoniumchloride (CTAC) as a structure-directing agent. In brief, $1.19 \mathrm{~g}$ of tetramethoxysilane (TMOS) was mixed with aminopropyl trimethoxysilane (APTMS) and FITC to create inherently fluorescent particles, and added to an alkaline solution containing the structure-directing agent cetyltrimethyl ammonium chloride (CTAC). The resulting synthesis mixture had a molar ratio of 0.9 TMOS: 0.1 APTMS: 1.27 CTAC: $0.26 \mathrm{NaOH}: 1439 \mathrm{MeOH}: 2560 \mathrm{H}_{2} \mathrm{O}$. The suspension was stirred overnight at room temperature (RT). The particles were separated by centrifugation, washed with deionized water and dried under vacuum for $24 \mathrm{~h}$. The structure-directing agent was then removed by ultrasonication in acidic ( $\mathrm{HCl}$ ) ethanol three times. These particles were PEI-functionalized by hyperbranching surface polymerization to yield sample $\mathrm{PEI}_{\text {graft }} \mathrm{MSN}_{2} \mathrm{~S}[17-$ 19].

For PEGylation, $\mathrm{MSN}_{2} \mathrm{~S}$ and $\mathrm{PEI}_{\text {graft- }} \mathrm{MSN}_{2} \mathrm{~S}$ were dispersed in chloroform and allowed to react with HMDI (4,4'methylenebis (cyclohexyl isocyanate))- activated $5 \mathrm{kDa}$ methyl ether of PEG (mPEG) in the presence of $N, N$ Diisopropylethylamine (DIPEA) overnight at $60^{\circ} \mathrm{C}$ under reflux. After the reaction, the particles were washed with ethanol and vacuum dried to yield PEG-MSN ${ }_{2} \mathrm{~S}$ and PEG$\mathrm{PEI}_{\text {graft }} \mathrm{MSN}_{2} \mathrm{~S}$. Succinylation was performed according to a previously published protocol with minor modifications [19]. $\mathrm{MSN}_{2} \mathrm{~S}$ and $\mathrm{PEI}_{\text {graft }}-\mathrm{MSN}_{2} \mathrm{~S}$ were suspended in dimethylformamide (DMF) and succinic anhydride was added in excess and sonicated. The suspension was stirred overnight at RT, filtered, washed with ethanol and dried in vacuum at RT to yield Succ- $\mathrm{MSN}_{2} \mathrm{~S}$ and Succ$\mathrm{PEI}_{\text {graft }} \mathrm{MSN}_{2} \mathrm{~S}$.

\subsection{Characterization of the fluorescence properties of MSNs}

\subsubsection{Determination of incorporated fluorophore amount and the fluorescence intensity of mesoporous silica nanoparticles}

The quantification of incorporated/leached amount of fluorophores for the synthesized MSNs was carried out by 
spectroscopic measurements. The absorption spectra of fluorophores was recorded on a Nanodrop2000c UV-VIS spectrophotometer with the help of standard curves prepared at different concentrations as given in Supplementary Figure S1. The concentration values of fluorophores was calculated for FITC and TRITC at the absorbance wavelengths $492 \mathrm{~nm}$ and $547 \mathrm{~nm}$, respectively.

The fluorescence intensity of MSN suspensions were determined by fluorescence spectrometry (Perkin Elmer LS 50B, PerkinElmer, Waltham, MA, USA) by dispersing them in various media (HEPES buffer at $\mathrm{pH}$ 7.2, MES buffer at pH 5 and Dulbecco's Modified Eagle's Medium, DMEM, $\mathrm{pH} 7.4$ ) at a concentration of $0.5 \mathrm{mg} / \mathrm{ml}$. The spectra were recorded by excitation wavelength $490 \mathrm{~nm}$ for $\mathrm{F}-\mathrm{MSN}_{1} \mathrm{~S}$ and $\mathrm{PEI}_{a d s}-\mathrm{F}_{-\mathrm{MSN}} \mathrm{S}$, $555 \mathrm{~nm}$ for T-MSN ${ }_{1}$ S and $\mathrm{PEI}_{a d s} \mathrm{~T}-$ $\mathrm{MSN}_{1}$ samples. The fluorescence spectra of samples were recorded for each media.

\subsubsection{Investigation of the structural alteration upon dissolution of MSNs}

The alteration in the porous order of $\mathrm{MSN}_{1} \mathrm{~S}$ were followed over time after redispersing them with the aid of ultra-sonication and incubating in HEPES buffer as well as ethanol at a concentration $0.5 \mathrm{mg} / \mathrm{mL}$ in parallel. Beside this, the porous order change during PEI coating and after long-term incubation of F-MSN $\mathrm{S}_{1}$ sere investigated. This part of the study was carried out by powder XRD, utilizing a Kratky compact small-angle system (MBraun, Nottinghampshire, UK).

\subsubsection{Investigation of fluorophore leaching from labeled MSNs}

Non-fluorescent $\mathrm{MSN}_{1}(\mathrm{MSN})$, fluorophore incorporated $\mathrm{MSN}_{1}\left(\mathrm{~F}-\mathrm{MSN}_{1}, \mathrm{~T}-\mathrm{MSN}_{1}\right)$ and PEI coated fluorophore incorporated $\mathrm{MSN}_{1} \mathrm{~S}\left(\mathrm{PEI}_{a d s}-\mathrm{F}-\mathrm{MSN}_{1}, \mathrm{PEI}_{a d s}-\mathrm{T}-\mathrm{MSN}_{1}\right)$ were dispersed in HEPES buffer at $0.5 \mathrm{mg} / \mathrm{mL}$ concentration and left for incubation at $37^{\circ} \mathrm{C}$ under gentle shaking. At each time point, prepared $2 \mathrm{~mL} \mathrm{MSN}{ }_{1}$ dispersion was taken out, centrifuged at $10510 \mathrm{~g}$ for 10 minutes and the supernatant was collected to determine the leached out fluorophore concentration by spectroscopic measurements. In this part of the study, non-fluorescent $\mathrm{MSN}_{1}$ s were used as a background for the measurements. After centrifugation, the cake of F-MSN $1 \mathrm{~S}$ and T-MSN $\mathrm{M}_{1}$ were collected in order to cross-check the remaining fluorophore content by dissolving the particles in $2 \mathrm{M} \mathrm{NaOH}$ solution and reading the sam- ple solutions by UV-VIS spectrophotometer to determine the remaining amount of fluorophore in the MSNs.

\subsection{Photostability measurements}

\subsubsection{Cell culturing}

HeLa cells (Human cervical adenocarcinoma) were grown in DMEM growth medium $(10 \% \mathrm{FCS}, 1 \%$ amino acids, $1 \%$ penicillin-streptomycin) at $60-70 \%$ confluency on coverslips. $10 \mu \mathrm{g} / \mathrm{ml}$ of a) F-MSN ${ }_{1}$, b) $\mathrm{PEI}_{a d s}-\mathrm{F}_{-} \mathrm{MSN}_{1}$, c) equivalent amount of FITC free dye, d) T-MSN ${ }_{1}$ e) $\mathrm{PEI}_{a d s}-\mathrm{T}^{-M_{S N}}$ and $\mathrm{f}$ ) equivalent amount of TRITC free dye was prepared in $1 \mathrm{ml}$ of cell growth media. The cell media containing particles was added to cells growing on coverslips. After 6 hours of incubation, the medium was removed and cells were washed twice with media and once with PBS. Cells were then fixed with 4\% paraformaldehyde in PBS, $\mathrm{pH} 7.4$ (PFA) for 10min at RT and further washed 3 times with PBS and mounted with prolong antifade reagent (Life Technologies) on cover slips for imaging.

\subsubsection{Photostability analysis and cell imaging by confocal microscopy in HeLa cells}

Confocal microscopy was performed using TCS SP5 STED (Leica Microsystems), LASAF software (Leica application suite), and 100X oil objectives. An Argon laser at 488nm (5.5 $\mu \mathrm{W} / \mu \mathrm{m}^{2}$ power focus) was used for excitation of FITClabeled samples and Diode laser (DPSS) $561 \mathrm{~nm}\left(6 \mu \mathrm{W} / \mu \mathrm{m}^{2}\right.$ power output) was used for excitation of TRITC-labeled samples. The emission from FITC-labeled particles was collected by HyD at 500-550 nm and TRITC emission was collected by PMT at $570-620 \mathrm{~nm}$. To measure the photostability of fluorophores conjugated to MSNs inside cells, regions of interest (ROI) were chosen inside endosomes and ROIs were allowed to bleach by using high laser power for each ROI. The intensity decay from ROI due to photobleaching was measured by fitting a single exponential decay curve.

\subsubsection{Photostability measurements in cell lysate}

HeLa cells lysates were prepared using lysis buffer. $10 \mu \mathrm{g}$ MSNs per mL of cell lysate were prepared for a) $\mathrm{F}-\mathrm{MSN}_{1} \mathrm{~s}$, b) equivalent amount of FITC free dye, c) T-MSN ${ }_{1} \mathrm{~S}$ and d) equivalent amount of TRITC free dye. $10 \mu \mathrm{l}$ of each sample was added to a 96 well glass bottom plates (Ibidi) and $50 \mu \mathrm{l}$ 
of mowiol was added on the top. The samples were allowed to dry overnight.

\subsubsection{In vivo imaging of PEG-PEI-T-MSNs in CAM model}

The in vivo imaging of PEG-PEI-T-MSNs was performed in a chick embryo chorioallantoic membrane (CAM) model. The CAM model provides technical simplicity of an in vivo environment and its optical transparency makes it a suitable animal model for optical in vivo two-photon imaging, and we have also recently established good correlation between CAM and murine models used for the evaluation of MSNs [20]. The experimental setup consisted of TCS SP5 MP (Multi-photon, Leica microsystems), LASAF software (Leica application suite), Non-descanned detectors (NDD) and 20X dip objectives. Two-photon microscopy was performed with Ti-sapphire femtosecond pulse at $800 \mathrm{~nm}$ for detectability of PEG-PEI-T-MSNs in blood vessels of live CAM placed on pre-warmed gel packs at $37^{\circ} \mathrm{C}$. A master mix consisting of $450 \mu \mathrm{g} / \mathrm{ml}$ of FITC dextran, $1.35 \mathrm{mg} / \mathrm{ml}$ of PEG-PEI-T-MSNs, and 90 uM Hoechst nuclear dye was prepared for injection. The $50 \mathrm{ul}$ volume of master mix was injected in one of the principal blood vessels of CAM for detectability of MSN particles as stable in vivo probes. The emission from the particles was collected using NDD detectors (NDD1-3) at 430-480 nm (Hoechst-nuclei), 500$550 \mathrm{~nm}$ (FITC dextran-blood vessels) and 565-605 nm (PEG-PEI-T-MSNs) respectively.

\subsection{In vitro characterization of surface modified MSNs}

\subsubsection{Cell lines}

Caco-2 (Human colorectal adenocarcinoma) cells and HeLa (Human cervical adenocarcinoma) cells obtained from ATCC (Manassas, VA, USA) were maintained in DMEM medium (Sigma, St. Louis, MO, USA) supplemented with $10 \%$ fetal calf serum (BioClear, Wiltshire, UK), $2 \mathrm{mM} \mathrm{L-}$ glutamine, $100 \mathrm{U} / \mathrm{ml}$ penicillin, $100 \mu \mathrm{g} / \mathrm{ml}$ streptomycin at $37^{\circ} \mathrm{C}$ in a $5 \% \mathrm{CO}_{2} / 95 \% \mathrm{O}_{2}$ and $90 \% \mathrm{RH}$ humidify atmosphere and handled under sterile conditions.

\subsubsection{In vitro cytotoxicity by WST-1 assay}

The WST-1 cell proliferation assay is based on the cleavage of the tetrazolium salt WST-1 to formazan by mitochondrial dehydrogenase enzyme, where the amount of formazan dye formed directly correlates to the number of metabolically active cells in the culture. HeLa cells were transferred to 96-well plates (6000 cells/well) and allowed to attach and grow. After $24 \mathrm{~h}$, the medium was removed and replaced with $100 \mu \mathrm{l}$ medium containing different concentrations of MSNs $(10,25 \mu \mathrm{g} / \mathrm{ml})$ for $72 \mathrm{~h}$. After incubation, $10 \mu$ l of WST-1 reagent (Roche Applied Science, Upper Bavaria, Germany) was added to each well and the plate was further incubated for $90 \mathrm{~min}$ at $37^{\circ} \mathrm{C}$ in $5 \% \mathrm{CO}_{2}$. After incubation with WST-1 reagent, the absorbance of the colored formazan was measured at $430 \mathrm{~nm}$ wavelength using Varioskan microplate reader (Thermo Scientific, Logan UT, USA) to determine cell viability.

\subsubsection{Intracellular uptake studies by flow cytometry}

Cells were seeded into 12-well plate and incubated for $24 \mathrm{~h}$ at $37^{\circ} \mathrm{C}$. The $65-70 \%$ confluent cells were then incubated with different FITC-labeled $\mathrm{MSN}_{2} \mathrm{~S}$ at $5 \mu \mathrm{g} / \mathrm{mL}$ for $4 \mathrm{~h}$. After incubation with $\mathrm{MSN}_{2} \mathrm{~S}$, the cells were trypsinized and the extracellular fluorescence was quenched by resuspension in trypan blue $(200 \mathrm{mg} / \mathrm{mL})$ for $5-10 \mathrm{~min}$ at room temperature. Thereafter, the cells were washed and resuspended in $0.4 \mathrm{~mL}$ phosphate-buffered saline (PBS). The amount of endocytosed particles inside cells was analyzed by BD FACSCalibur flow cytometer (FL-1, BD Pharmingen). The mean fluorescence intensity (MFI) of the cells at FL-1 channel was measured. The data was analyzed with BD Cel1Quest Pro ${ }^{\mathrm{TM}}$ software for total amount of MSNs uptake by 10000 cells. GraphPad Prism 5.0 software was used for the statistical analysis of the results.

To study the uptake mechanism of different surface charged $\mathrm{MSN}_{2} \mathrm{~S}$ (PEI-, PEG-PEI-, Succ-PEI- MSNs), Caco2 cells were incubated with various pharmacological inhibitors, including Sodium azide (10 mM), Amiloride $(25 \mu \mathrm{M})$, Genistein $(200 \mu \mathrm{M})$ and Phenyl arsine oxide $(1 \mu \mathrm{M})$ for $1 \mathrm{~h}$ and then cells were further incubated with MSNs for $4 \mathrm{~h}$. The amount of endocytosed particles was analyzed by flow cytometery.

\subsubsection{Uptake studies by confocal scanning microscopy}

HeLa cells were seeded in 12-well plates containing cover slips, one day before treatment, and the cells were treated with $5 \mu \mathrm{g} / \mathrm{mL}$ of MSNs for $4 \mathrm{~h}$. After the treatment, the cells cultured on cover slips were washed with PBS and labeled with rhodamine-lectin (Vector laboratories, USA) for $15 \mathrm{~min}$ at $37^{\circ} \mathrm{C}$. Then cells were fixed with 4\% PFA for $10 \mathrm{~min}$ at room temperature, and 
mounted with DAPI (Vectashield; Vector Laboratories Inc, Burlingame, CA). The intracellular localization of MSNs was viewed and imaged under a confocal laser-scanning microscope (LSM 510 META, Carl Zeiss Inc., Thornwood, $\mathrm{NY}$ ) equipped with an argon laser using (63X oil objective, $405 \mathrm{~nm} / 488 \mathrm{~nm} / 543 \mathrm{~nm}$ excitation and 420-480 BP/500$520 \mathrm{~nm} \mathrm{BP/560LP} \mathrm{emission} \mathrm{filter).}$

\subsubsection{Protein adsorption studies}

For the protein adsorption studies, $\mathrm{MSN}_{2} \mathrm{~S}$ at concentrations of 0.5 and $1 \mathrm{mg} / \mathrm{mL}$ were dispersed in 1\% FCS (Fetal Calf Serum) in HEPES buffer for $4 \mathrm{~h}$. The initial protein concentration of $1 \%$ FCS was measured by spectrophotometry at the wavelength $285 \mathrm{~nm}$. After the incubation period, the particles were centrifuged and the supernatant was collected. The concentration of protein in the supernatant was measured, and the amount of protein adsorbed on the particle surface was obtained by subtracting the protein concentration in the supernatant from the initial protein concentration.

To investigate the effect of protein adsorption on the zeta potential, $\mathrm{MSN}_{2} \mathrm{~S}$ were first dispersed at $2 \mathrm{mg} / \mathrm{mL}$ in HEPES buffer as a stock. Subsequently, the particles were dispersed in DMEM at $200 \mu \mathrm{g} / \mathrm{mL}$ and incubated for $2 \mathrm{~h}$ at $37^{\circ} \mathrm{C}$. The particles were centrifuged and redispersed in HEPES buffer, whereby the zeta potential was measured.

\section{Results and Discussion}

For our observations to be as generally applicable as possible, two of the most frequently employed MSN platforms were chosen for the demonstrations: pure silica MSNs and amino-functionalized MSNs prepared via cocondensation. All MSN particles used in this study were prepared according to our earlier studies $[14,16]$ and were, accordingly, of spherical shape with radially aligned pores (Figure 1). The particles were prepared via two slightly differing procedures, in order to yield either negatively charged $\left(\mathrm{MSN}_{1}\right)$, representing that of pristine silica, or close-to neutral amino-co-condensed $\left(\mathrm{MSN}_{2}\right)$ starting particles. In the latter case, 10\% aminopropylsilane was used as silica source in order to create an abundance of primary amino groups on the resulting MSN surface for allowing further functionalization; whereas in the former case the inherent negative charge of silica surfaces can be exploited to enable electrostatic adsorption of poly(ethylene imine), PEI. In this case, only enough aminopropylsilane was used

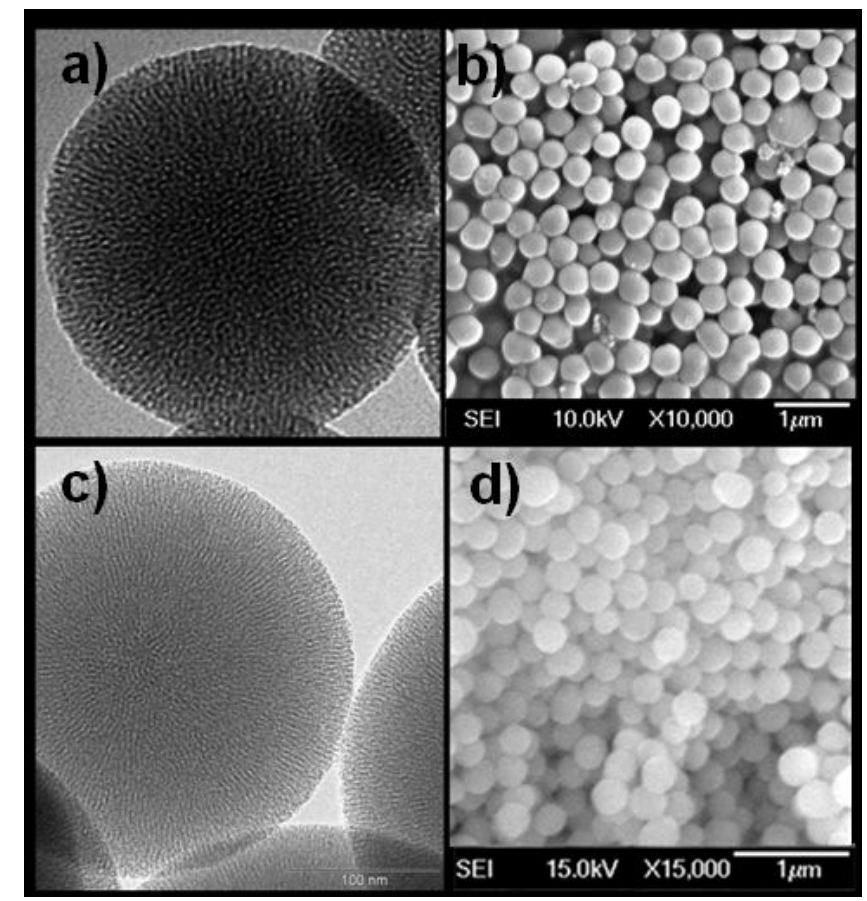

Fig. 1. Typical (a) TEM and (b) SEM image of the $M \mathrm{MN}_{1}$ series. Typical (c) TEM and (d) SEM image of the $M \mathrm{SN}_{2}$ series.

to allow for the co-condensation of previously formed DyeAPTES conjugates, to yield fluorescent MSNs with physicochemical characteristics still resembling those of pristine silica. The physicochemical characteristics of both MSN series have been summarized in Table 1 .

\subsection{Influence of the fluorescent label on the fluorescence properties of the MSNs}

In order for MSNs to be studied in a biological setting, they are typically fluorescently labeled. Since most fluorescent tags are available as amine-reactive conjugates, MSNs can very conveniently be labeled by pre-conjugation of the fluorescent label/dye with aminosilanes with subsequent reaction of the fluorescently tagged silane with the MSN either by incorporation already in the synthesis step via co-condensation, or via post-grafting methods. The particles in the present study were all prepared via the co-condensation method. The pre-eminently most frequently applied fluorescent label is fluorescein, most commonly used in the 'activated' form of fluorescein isothiocyanate (FITC) where the ITC is an amine-reactive group. However, fluorescein is a pH-probe [23, 24], which essentially means that the fluorescence intensity will depend on the surrounding $\mathrm{pH}$ during detection. This is highly relevant for cellular studies, whereby the $\mathrm{pH}$ between differ- 
Table 1. Physico-chemical characterization of MSN particle suspensions in aqueous solvent (HEPES buffer, $\mathrm{pH}=7.2)$ and determination of the surface modification degree by thermogravimetrical analysis. F=FITC and T=TRITC dye. MSN 2 series are all FITC-labeled. $\lambda_{e x}=490 \mathrm{~nm}$ for FITC- and $555 \mathrm{~nm}$ for TRITC-conjugated MSNs.

*) the last modification step only considered.

\begin{tabular}{|c|c|c|c|}
\hline Type of Particle & $\zeta$-potential $[\mathrm{mV}] \mathrm{pH}=7.2$ & Fluorescence Intensity $\lambda=$ max. peak & Organic Portion added* [wt\%] \\
\hline F-MSN 1 & -34 & 973 & - \\
\hline T-MSN 1 & -27 & 876 & - \\
\hline $\mathrm{PEI}_{\text {ads }}-\mathrm{F}-\mathrm{MSN}_{1}$ & +52 & 340 & $\sim 10$ \\
\hline $\mathrm{PEI}_{\mathrm{ads}}-\mathrm{T}-\mathrm{MSN}_{1}$ & +48 & 207 & $\sim 10$ \\
\hline $\mathrm{MSN}_{2}$ & +5 & 895 & - \\
\hline $\mathrm{PEI}_{\text {graft }}-\mathrm{MSN}_{2}$ & +56 & 193 & $\sim 10$ \\
\hline PEG-MSN 2 & +1 & 867 & $\sim 9.7$ \\
\hline PEG-PEI graft $-\mathrm{MSN}_{2}$ & +15 & 163 & $\sim 3.2$ \\
\hline Succ-MSN $_{2}$ & -20 & 102 & $\sim 4.3$ \\
\hline Succ-PEI ${ }_{\text {graft }}-\mathrm{MSN}_{2}$ & -65 & 95 & $\sim 5.9$ \\
\hline
\end{tabular}

ent intracellular compartments vary from acidic (around $\mathrm{pH}$ 5) to neutral ( $\mathrm{pH} 7$ ). By theory, the quantum yield between acidic and basic $\mathrm{pH}$ for fluorescein varies with a factor of three, so the difference is substantial when quantifications based on fluorescence are attempted for; such as cellular uptake studies by flow cytometry/fluorescenceassisted cell sorting, image analysis by fluorescence confocal microscopy etc. Fluorescein can adopt many chemical forms, of which only two are fluorescent - the monoanion and dianion, with quantum yields of 0.37 and 0.93 , respectively [23]. Depending on the preparation conditions (MSN synthesis, template extraction, surface functionalization, washing and separation steps, etc.) the MSN-conjugated fluorophore can thus be exposed to conditions (solvents, $\mathrm{pH})$ that induces one of its non-fluorescent forms. Consequently, correlation between fluorescence intensity and amount of fluorophore, frequently used to represent the amount of particles, is highly prone to uncertainties; except under tightly controlled conditions. Further, attachment/incorporation of the fluorophore to a solid support, such as a particle, may also induce changes in its local environment [21, 22] leading to alterations in their fluorescence properties. This is especially true for MSNs, where the major portion of the fluorophores (given being prepared via the abovementioned strategies) would be located in a confined space like the mesopore, where the local environment such as $\mathrm{pH}$ inside the pores are not necessarily matching with those of the bulk solvent (surrounding) conditions [25]. This is due to the acidity of the silanols, which are always present on a silica surface. Adding basic groups, such as aminosilanes used to conjugate the fluorophore, thus adds to this complexity. Employing surface coatings with acid/base properties in- evitably increases the level of complexity even more, especially when it comes to the inner versus the outer surface juxtaposition.

Poly(ethylene imine), PEI, a polycation with the highest positive charge density [26] is a commonly employed polymer in nanomedical context, due to a number of beneficiary properties such as: a) providing a high density of reactive groups for attachment of active molecules, owing to the high concentration of terminal amine groups originating from the hyperbranched structure; b) providing high particle suspension stability via electrostatic stabilization; c) enabling surface charge tuning via derivatization or even 'charge capping', which can be utilized to suppress unspecific biointeractions; d) promoting endosomal escape via its characteristic "proton sponge" or "endosomal buffering" ability [27], and even potentially e) providing 'molecular gate' properties to porous carriers by adopting different conformations at different pHs. The enormous buffering capacity imparted by the high abundance of amines - primary, secondary, and tertiary all with their own pKa values, also suggests that the local $\mathrm{pH}$ experienced by attached molecules on a particle with such a coating could be affected to a great degree. To investigate this potential effect versus the influence of the surrounding media, FITC-conjugated MSNs $\left(\mathrm{F}-\mathrm{MSN}_{1} \mathrm{~s}\right)$ were compared to that of similar MSNs where FITC had been exchanged to TRITC (T-MSN ${ }_{1}$ s), an ITC-activated rhodamine dye. Due to the claimed $\mathrm{pH}$-insensitivity of rhodamine dyes as compared to fluorescein, these two dyes have even been used as pairs in intracellular ratiometric $\mathrm{pH}$ sensing, as also previously demonstrated for MSN systems [28]. 


\subsubsection{Influence of the surrounding media}

Fluorescent labels FITC and TRITC were thus incorporated into $\mathrm{MSN}_{1}$ to yield F-MSN ${ }_{1}$ S and T-MSN ${ }_{1}$ s, respectively, via the co-condensation method. The produced fluorescent particles (without additional surface functionalization) were dispersed in buffer solutions of cellular relevance, i.e. MES buffer ( $\mathrm{pH} 5$ corresponding to endo/lysosomal $\mathrm{pH}$ ), HEPES buffer ( $\mathrm{pH} 7.2$ corresponding to cytoplasmic $\mathrm{pH}$ ) as well as serum-supplemented DMEM cell media (Figure 2). Clearly, the fluorescence of the FITC-conjugated MSNs exhibited significantly lower fluorescence at acidic $\mathrm{pH}$ than neutral, as expected; while no difference in fluorescence behavior was found for the TRITC-conjugated counterparts in the two different buffer solutions. Thus, this was in perfect agreement with the well-established behavior of the free dyes. The fluorescence behavior in DMEM, however, seemed to have an opposite effect on both particle types, with strongly enhanced fluorescence observed for the F$\mathrm{MSN}_{1}$ but slightly decreased fluorescence for the T-MSN 1 . Currently we have no conclusive explanation for this behavior, but note as an important consequence that since MSNs are incubated in cell media for in vitro studies, quantification attempts based on measurements made under less-biological conditions may not apply when extrapolated to biological conditions. The composition of cell media is quite complex, and the exact reason may thus be hard to isolate. Biologicals do exhibit autofluorescence to some degree, and the cell media contains serum; these components may adsorb to the particles to a different degree which may induce either enhancement or quenching of the observed fluorescence [29]. The pH-dependent effect was, however, in this case clear-cut and in accordance to the expected behaviors of the free dyes.

\subsubsection{Influence of the surface coating}

Given this pH-dependent behavior, which was in agreement with the free dye behavior, the fluorescence emission of the unmodified particle suspensions could supposedly also be used as "standard curves" to probe the lo$\mathrm{cal} \mathrm{pH}$ that the fluorescein molecules are experiencing on corresponding surface-modified MSNs. Thus, the fluorescence spectra were recorded for the unmodified F-MSN ${ }_{1} \mathrm{~S}$ at a range of different pHs (Figure 3a) and the intensity at the maximum peak was plotted against $\mathrm{pH}$ (Supplementary Figure S2). Subsequently, commercial hyperbranched PEI (25 kDa) was adsorbed to the (same) particles via electrostatic adsorption at neutral $\mathrm{pH}$ (HEPES buffer, $\mathrm{pH}$ 7.2) whereafter the particles were separated, washed, dried

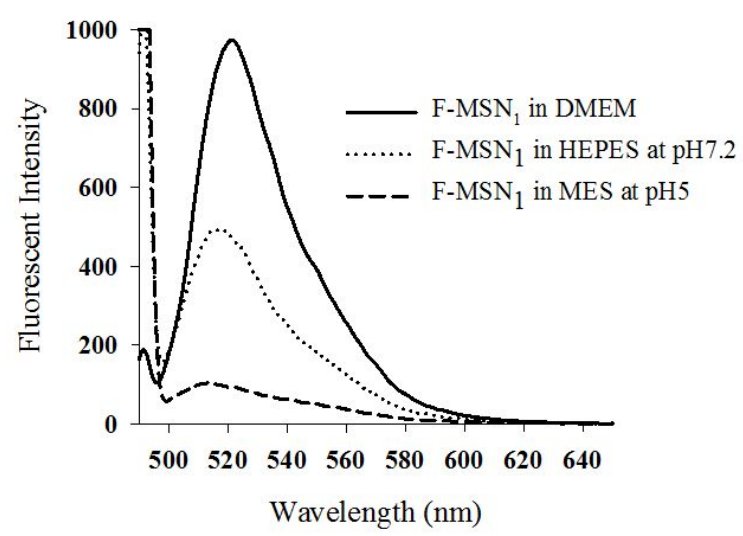

(a)

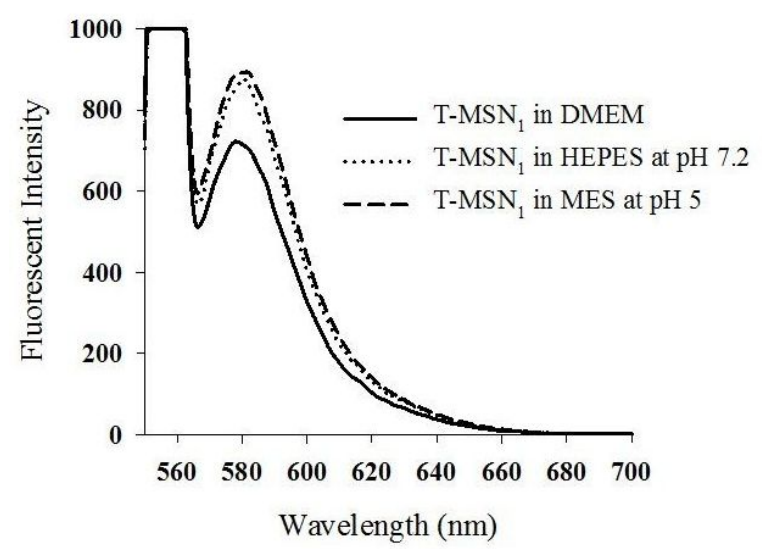

(b)

Fig. 2. Fluorescence spectra of $F-M S N_{1}$ and T-MSN ${ }_{1}$ at neutral and weakly acidic pH buffer solutions as well as in cell media(DMEM). $\lambda_{e x}=490 \mathrm{~nm}$ for FITC- and $555 \mathrm{~nm}$ for TRITC-conjugated MSNs.

and redispersed in ion-exchanged water. The fluorescence intensity was matched to that of the standard curve to find the $\mathrm{pH}$ which, in this sense, was ascribed as the "local $\mathrm{pH}$ " experienced by the fluorescein molecules of the surfacemodified $\mathrm{MSN}_{1} \mathrm{~S}$ and, in this case, was very close to the $\mathrm{pH}$ value of 6 . It is noteworthy that a change in solvent $\mathrm{pH}$ did not induce any difference in fluorescence intensity for the PEI-modified MSNs (see also Figure 4), which supports the fact that it is, indeed, the "local $\mathrm{pH}$ " being probed. We ascribe this phenomenon to the well-known buffering capacity of PEI [30], which in this case was shown to be strong enough to overcome the influence of the surrounding 'environmental' $\mathrm{pH}$ for PEI-modified MSNs. To further investigate whether the structure of the PEI surface coating could have an influence, we compared these $\mathrm{PEI}_{a d s}-\mathrm{F}_{-} \mathrm{MSN}_{1}$ particles to those that had been modified by surface hyperbranching polymerization of PEI, PEI graft $^{-}$ 

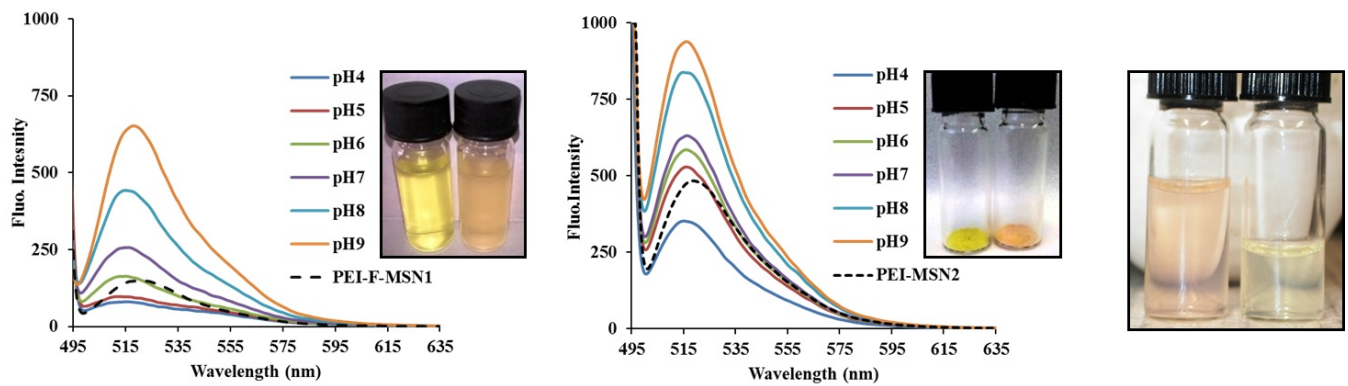

(a)

Fig. 3. Dependence of FITC-conjugated MSN fluorescence intensity as a function of $\mathrm{pH}$. a) F-MSN $\mathrm{M}_{1}$ fluorescence intensity variability with solvent $\mathrm{pH}$. Coating with PEI (10 wt\%) via electrostatic adsorption results in the same emission spectra regardless of solvent $\mathrm{pH}$. Inset: $\mathrm{F}-\mathrm{MSN}_{1} \mathrm{~s}$ (left) vs $\mathrm{PEI}_{a d s}-\mathrm{F}-\mathrm{MSN}_{1} \mathrm{~s}$ (right) in HEPES buffer. b) Same as previous repeated for $\mathrm{MSN}_{2}$ and surface-grafted $\mathrm{PEI}_{\text {graft }}-\mathrm{MSN}_{2}$, resulting in an enhanced

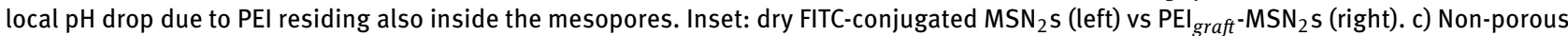
(Stöber) control particle. On the left $\mathrm{PEI}_{\text {graft }}-\mathrm{SiO}_{2}\left(+39 \mathrm{mV}\right.$ surface charge, $\left.\lambda_{\max , A b s}=501.5 \mathrm{~nm}, \mathrm{I}_{\text {max } \text {,fluo }}=174\right)$ and on the right the same particle but further succinylated $\left(-42 \mathrm{mV}\right.$ surface charge, $\left.\lambda_{\max , A b s}=488 \mathrm{~nm}, \mathrm{I}_{\max , \text { fluo }}=547\right)$ as measured in HEPES buffer at pH 7.2.

$\mathrm{MSN}_{2} \mathrm{~S}$ (see Table 1). Also here, the fluorescence intensity of the 'starting' (unmodified) FITC-labeled $\mathrm{MSN}_{2}$ particle was measured at different $\mathrm{pHs}$ to yield a similar standard curve as above, to which the $\mathrm{PEI}_{\text {graft }}$-MSN 2 intensity was thereafter compared (Figure 3b, Supplementary Figure S2). In this case, the local $\mathrm{pH}$ was found to be slightly below 5 . This result could very well be due to the fact that in the surface hyperbranching process, PEI is grown both on the inner (pore wall) and outer (particle) surfaces; whereas by adsorption of large-molecular $(25 \mathrm{kDa})$ weight PEI, the location of the PEI polymer would be predominantly on the outer particle surface. Thus, being present both on the outside particle surface and inner pore walls, the local "proton trap" effect of PEI would also be more predominant inside the pores in the case of grafted PEI, which is reflected in the lower local $\mathrm{pH}$ (i.e. higher local concentration of protons) observed. This distribution of amine groups also on the inner pore walls further imparts MSNs surface functionalized adopting this strategy with very efficient loading capacity of negatively charged hydrophilic guest molecules, such as acidic small-molecular compounds, for which $>40 \mathrm{wt} \%$ could be attained even under aqueous conditions due to the highly favorable electrostatic interactions [31]. The accompanied red shift observed for both the PEI-MSN peaks (Figure 3a,b) could be due to the thus induced local polarity change, brought upon by the local abundance of protons, similar to that of positive solvatochromism rather than a $\mathrm{pH}$-induced effect.

To confirm that the observed intensity decrease was not due to chemical alterations/reactions of the fluorophore molecule itself, but predominantly an effect of local $\mathrm{pH}$, we used a non-porous Stöber [32] silica particle $\left(\mathrm{SiO}_{2}\right)$ as control (Figure 3c) to eliminate the compli- cating factors induced by the porosity. Similarly as for the MSNs, PEI-functionalization (by surface hyperbranching polymerization in this case) induced a red shift in the absorbance, as evidenced by the similar orange color adopted as for the MSNs upon PEI-functionalization (Figure 3a,b insets). Thereafter, the PEI layer was capped with succinic acid groups ("succinylated") to yield terminal carboxylic acid groups instead of amines, which should counteract the PEI-induced local pH effect in terms of canceling out or suppressing the characteristic "proton trap" property. Quite correctly, the maximum fluorescence intensity increased approximately with a factor of three (from 174 for $\mathrm{PEI}_{\text {graft }}-\mathrm{SiO}_{2}$ to 547 for Succ-PEI ${ }_{\text {graft- }} \mathrm{SiO}_{2}$ ) upon succinylation, simultaneously confirming the reversibility of the observed intensity change.

To further confirm that no influence of the $\mathrm{pH}$ of the surrounding medium on the PEI-modified MSNs is taking place, the fluorescence was recorded for $\mathrm{PEI}_{a d s}-\mathrm{F}-\mathrm{MSN}_{1} \mathrm{~S}$ under the same conditions as in Figure 2. As can be seen also here (Figure 4), in the presence of a PEI coating, the surrounding $\mathrm{pH}$ has no effect on fluorescence intensity. Even the influence of cell media (DMEM)observed for the particles without a PEI coating above, is overridden by the strong influence of a PEI-coating (Figure 4a). Nevertheless, when comparing the fluorescence spectra of the $\mathrm{PEI}_{a d s^{-}}$ $\mathrm{F}-\mathrm{MSN}_{1}$ where the PEI was adsorbed in HEPES $\left(\mathrm{PEI}_{a d s}-\mathrm{F}-\right.$ $\left.\mathrm{MSN}_{1}-\mathrm{H}\right)$ to that in ethanol $\left(\mathrm{PEI}_{a d s}-\mathrm{F}-\mathrm{MSN}_{1}-\mathrm{E}\right.$, Figure $\left.4 \mathrm{~b}\right)$ the maximum peak values (intensities) do not match, despite being measured with the same settings and for the same starting particle. The clear difference in Figure 4a and Figure $4 \mathrm{~b}$ is intuitively ascribed to silica dissolution, as the PEI adsorption for $\mathrm{PEI}_{a d s}-\mathrm{F}-\mathrm{MSN}_{1}-\mathrm{H}$ was conducted in aqueous conditions overnight. During this time course, 


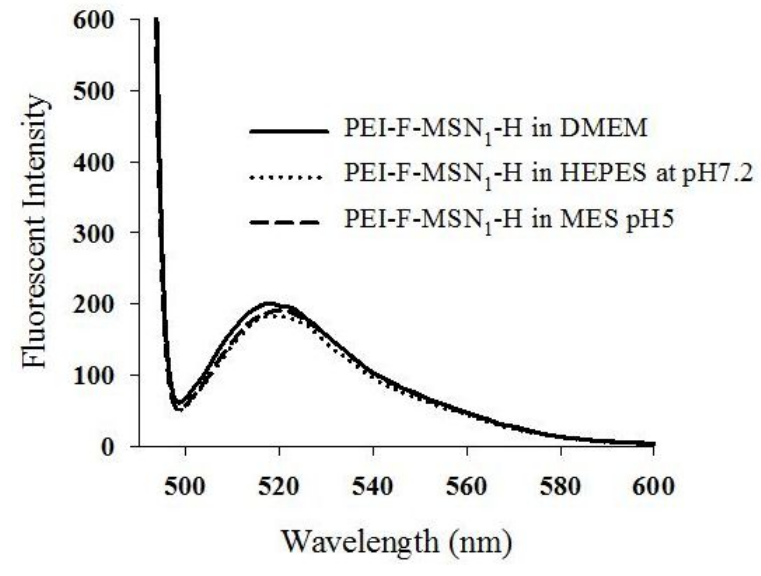

(a)

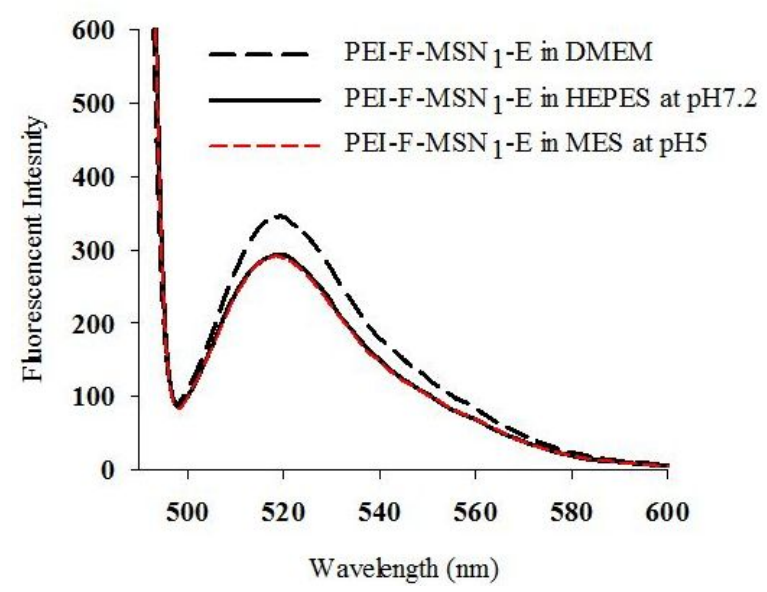

(b)

Fig. 4. Fluorescence spectra of PEI-coated $\mathrm{F}-\mathrm{MSN}_{1}$ at neutral and weakly acidic $\mathrm{pH}$ buffer solutions as well as in cell media (DMEM), where the PEl-adsorption has been conducted under different conditions (PEI-MSN $\mathrm{M}_{1}-\mathrm{H}$ in HEPES buffer; PEI-F-MSN $\mathrm{M}_{1}-\mathrm{E}$ in ethanol).

silica dissolution would be pronounced [33] and moreover, PEI could even catalyze the dissolution of silica, due to its high abundance of amine groups [34] (see also section 3.2).

\subsubsection{Influence of the particle concentration}

It is well known that fluorophores in close proximity to each other can lead to significant self-quenching effects $[35,36]$ and this is also where MSNs are expected to provide certain advantages over other particle designs, owing to the distribution of fluorophores throughout the porous network. In the current study, the amount of fluorophores was fixed; however, a similar observation could be expected if the particle concentration is high enough for the particles to be in close contact with each other.
This situation is prone to occur especially when nanoparticle uptake by cells is quantified when the majority of nanoparticles are in intracellular compartments such as endo/lysosomes as a result of endocytosis. To investigate whether particle concentration could have a profound effect on the fluorescence, we also measured the fluorescence intensity of F-MSN ${ }_{1}, \mathrm{PEI}_{a d s}-\mathrm{F}_{-} \mathrm{MSN}_{1}, \mathrm{~T}-\mathrm{MSN}_{1}$ and $\mathrm{PEI}_{a d s}-\mathrm{T}-\mathrm{MSN}_{1} \mathrm{~S}$ as a function of particle concentration (Figure 5a-d). Clearly, a remarkable effect of MSN concentration on fluorescence intensity is observed for all particles, where the concentration-dependence furthermore differs depending on the surrounding $\mathrm{pH}$ for fluorescein-labeled MSNs (Figure 5a). On the contrary, for the rhodamine-labeled particles (T-MSN ${ }_{1}$ and $\mathrm{PEI}_{a d s} \mathrm{~T}$ $\mathrm{MSN}_{1}$ ) a pH-independent behavior is evident, whereas a similar particle-concentration-dependent behavior can be observed. Since the settings of the instrument were adjusted for each separate measurement (graph) it is noteworthy that there was still a considerable effect of the PEIcoating of the T-MSN $\mathrm{M}_{1} \mathrm{~S}$ on the resulting fluorescence intensity (Supplementary Figure S3), which, however, based on the $\mathrm{pH}$-independency determined above (Figure $5 \mathrm{c}, \mathrm{d}$ ) should not be ascribed to a local $\mathrm{pH}$ effect as in the case of fluorescein. Here, the signal decrease could instead be due to a compound effect of non-radiative FRET, radiative reabsorption and scattering due to increased sample opacity upon addition of an organic surface coating.

\subsection{Influence of coating procedure on the dissolution of the MSNs}

In the case of utilizing pre-formed PEI polymers as surface coating, maximized electrostatic attraction is obtained under aqueous conditions around neutral $\mathrm{pH}$, when the silica is negatively charged due to deprotonation of silanols and PEI positively charged due to protonation of amines. Even though the electrostatic adsorption procedure of PEI was, in our case, carried out under concentrations $(3 \mathrm{mg} / \mathrm{mL})$ that are well above the solubility limit of silica in water at pH 7 (120 ppm) [37], dissolution and reprecipitation of silica can still readily take place under these conditions [38]. This should be most pronouncedly reflected in the mesoscopic ordering of the MSNs, whereby we applied smallangle X-ray diffraction to clearly illustrate this effect. First, $\mathrm{MSN}_{1} \mathrm{~S}$ were immersed in either ethanol or HEPES at $37^{\circ} \mathrm{C}$ and subject to shaking to replicate the coating conditions, and at different time points the sample was separated by centrifugation, dried, whereafter powder X-ray diffractograms were recorded (Figure 6). Clearly, no alteration in the mesoscopic order was observed after incubation in 

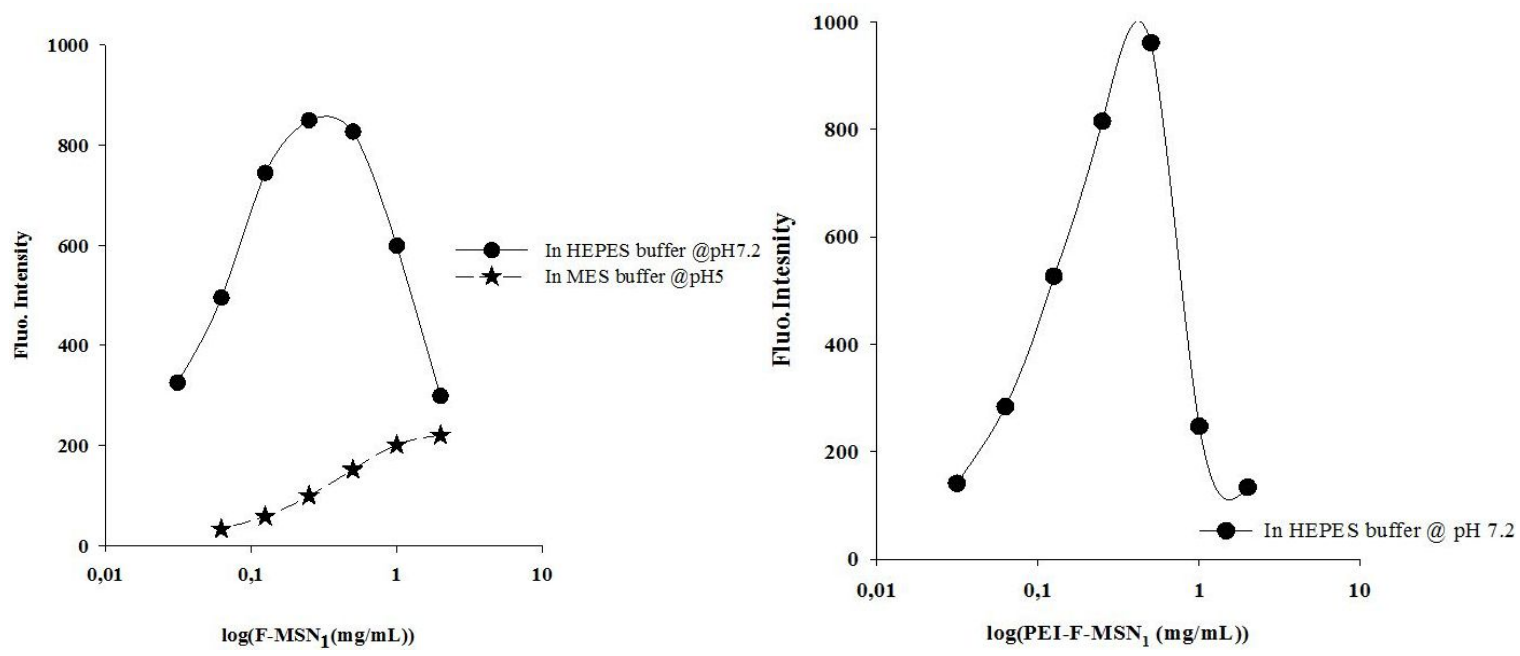

(a)

(b)
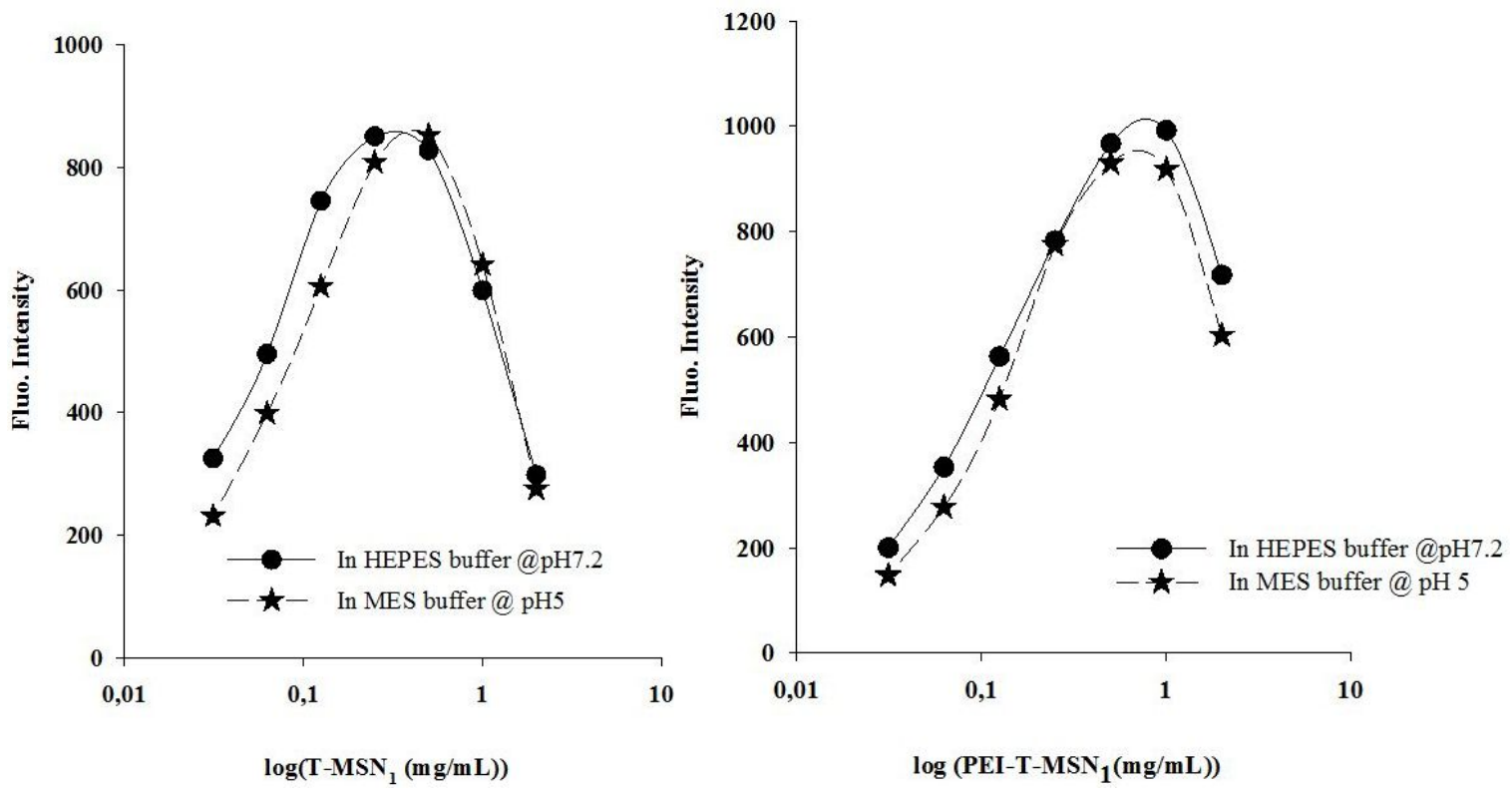

(c)

(d)

Fig. 5. Fluorescence intensity as a function of $M S N$ concentration for (a) F-MSN ${ }_{1} S$ (b) $P E I_{a d s}-\mathrm{F}-M S N_{1} S$ (c) $T-M S N_{1} S$ and (d) $P E I_{a d s}-T-M S N_{1} S$.

ethanol (Figure 6b), whereas complete structural collapse appeared to be the case for the sample subject to aqueous conditions already after $2 \mathrm{~h}$ of immersion (Figure 6a). Due to the radial alignment of these pores, the structural ordering is not that well-defined to begin with, but upon carrying out the synthesis at elevated temperatures $\left(80^{\circ} \mathrm{C}\right)$ a restructuring can take place and hexagonally ordered mesopores can be formed [15] (Figure $6 \mathrm{c}$,d solid lines). To more clearly deduce the structural effect by PXRD, such a sample was prepared and PEI-coating was performed under aqueous and ethanolic conditions, whereafter the diffractograms were recorded (Figure 6c,d). Similarly as above, the structural order was completely retained in ethanol, whereas only a slight 'bump' indicative of some remaining order was deducible for the $\mathrm{PEI}_{a d s}-\mathrm{F}-\mathrm{MSN}_{1}-\mathrm{H}$ coated under aqueous conditions (HEPES buffer). To confirm that, still, not complete disintegration is taking place owing to the aqueous conditions used, the unmodified MSNs prepared at $80^{\circ} \mathrm{C}$ were incubated for 10 days in HEPES at $37^{\circ} \mathrm{C}$ in a shaking water bath, whereafter the PXRD was measured. In this case, a more pronounced peak was still observable that could be assigned to the original $d_{100}$ peak for the hexagonally ordered phase; indicating that complete collapse of the structure does not take place even after sub- 


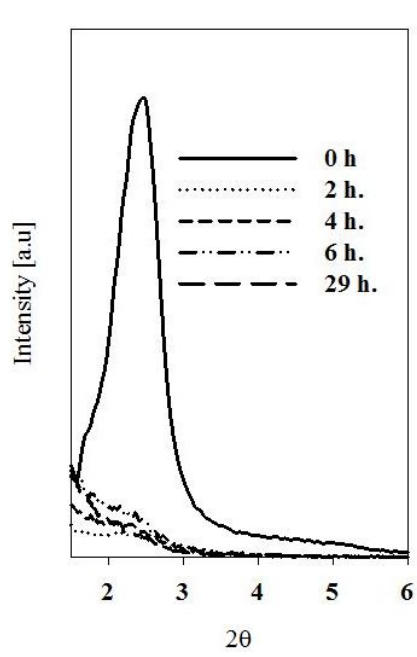

(a)

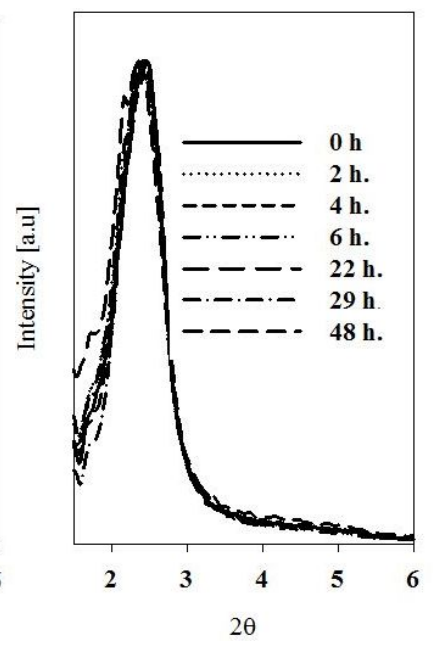

(b)

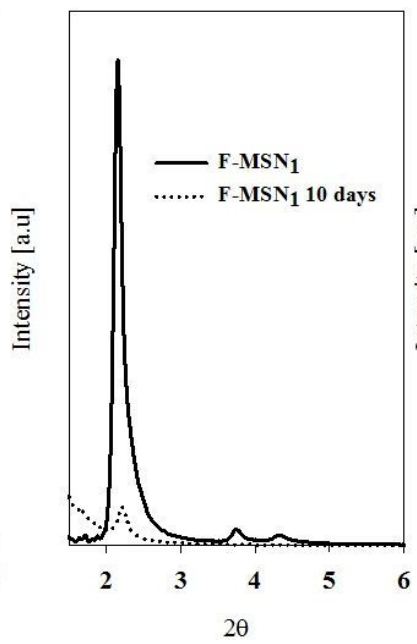

(c)

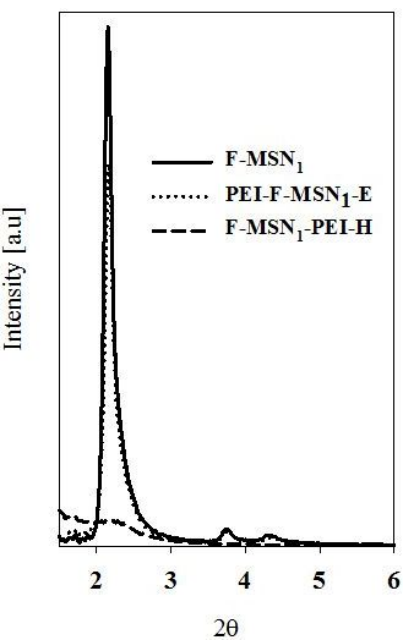

(d)

Fig. 6. Alteration of the mesostructural order of MSNs during incubation in ethanol and HEPES buffer at different time points. Pure MSN ${ }_{1}$

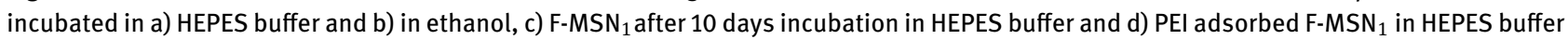
and in ethanol.

jected to these conditions for prolonged times. In this case, it should be noted that the retainment of the structural order may also be connected to the higher hydrolytic stability of MSNs synthesized under elevated temperatures. It should further be noted that a faster decrease in structural ordering in the presence of PEI can be expected due to the capability of amines to catalyze the dissolution of silica, as mentioned above. We do also not exclude the possibility of this effect being enhanced by the sonication treatment that usually accompanies the redispersion of particles in solution after drying, an effect of which may thus be accelerated by the presence of a catalyst [39]. It is, however, noteworthy that, after the leaching studies described below (involving one week of incubation in HEPES) both the morphological integrity as well as effective surface charge of the particles were also still retained to a certain degree, as judged by dynamic light scattering and electrokinetic measurements (Supplementary Figure S4).

\subsection{Influence of the MSN degradation on the leaching of attached labels}

As it is evident that the degradation (dissolution) of the material itself will have implications for any molecules that are attached to it, we also investigated the kinetics of fluorophore leaching under physiological conditions in terms of $\mathrm{pH}$ and temperature. Two different approaches were conducted: in the first case, the total amount of incorporated fluorophore was determined for all the $\mathrm{MSN}_{1} \mathrm{~S}$ by complete dissolution of the MSNs in $\mathrm{NaOH}$ and determination of the FITC/TRITC concentration spectrophotometrically. For all four MSNs studied here, the total amount of fluorophores was in comparable amounts of $\sim 1 \mathrm{wt} \%$. To avoid the obvious complications of fluorescence-based quantification, spectrophotometry was chosen over fluorimetry in this case. Thereafter, the $\mathrm{MSN}_{1} \mathrm{~S}$ were immersed in HEPES, and at given time points, the concentration of fluorophore in the supernatant was determined and compared to that of the original amount (Figure 7). Based on these results, it can be seen that for FITC, the leaching for the non-coated $\mathrm{MSN}_{1} \mathrm{~s}$ reaches around $\sim 50 \%$ already during the first 24 hours, after which it levels out and no change is observed for up to a week, which we tentatively ascribe to the static conditions used. For $\mathrm{PEI}_{a d s}-\mathrm{F}-\mathrm{MSN}_{1} \mathrm{~S}$ (Figure 7a), there is an initial burst that could be ascribed to the corresponding dissolution behavior in the presence of amines, whereafter a re-adsorption seems to be taking place. Intuitively this could be due to that the fluorescein molecule is negatively charged, and hence would desorb more easily from the negatively charged silica surface (unmodified MSNs) as opposed to the positively charged PEImodified surface. For T-MSN $\mathrm{N}_{1}$ (Figure $7 \mathrm{~b}$ ) the opposite behavior can be observed, which could be due to that the rhodamine molecule contains amines, so it would, in turn, be attracted to and adsorb on the negatively charged (unmodified) silica surface. Overall, a combined dissolutionreprecipitation (of silica) and adsorption-desorption (of fluorescent labels) equilibria is what is most likely being observed. This is supported by the notation that the hy- 


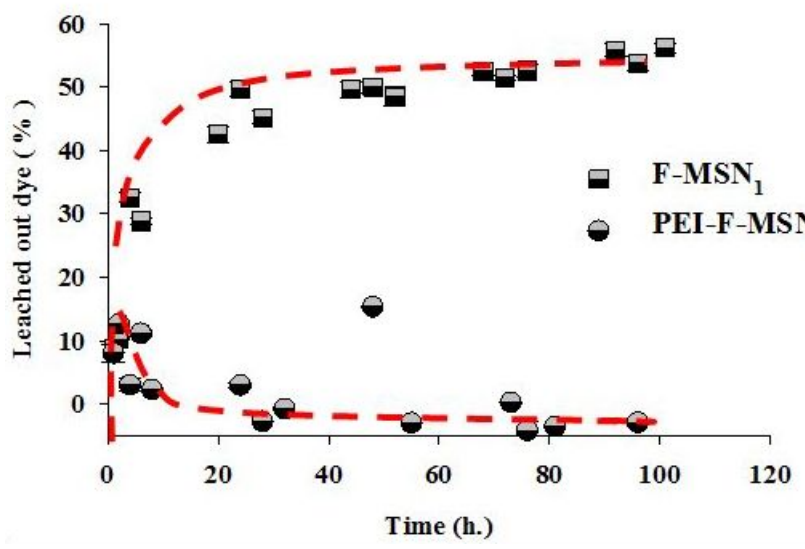

(a)

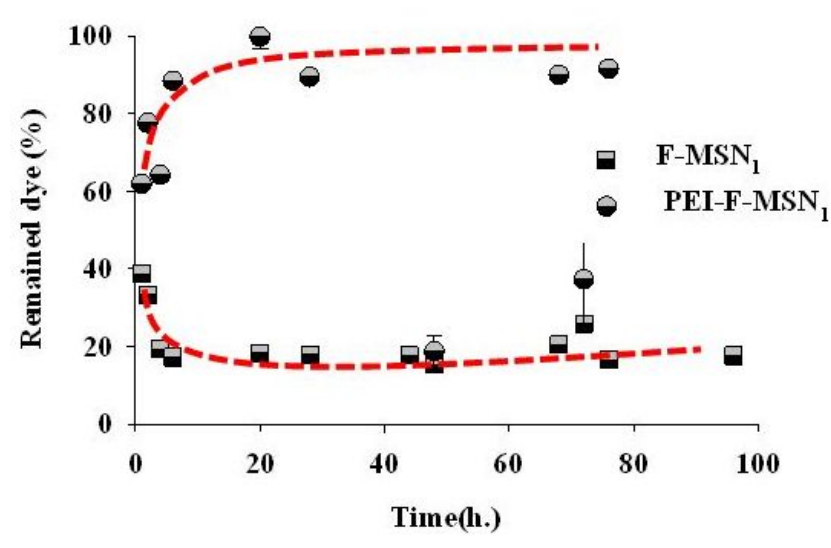

(c)

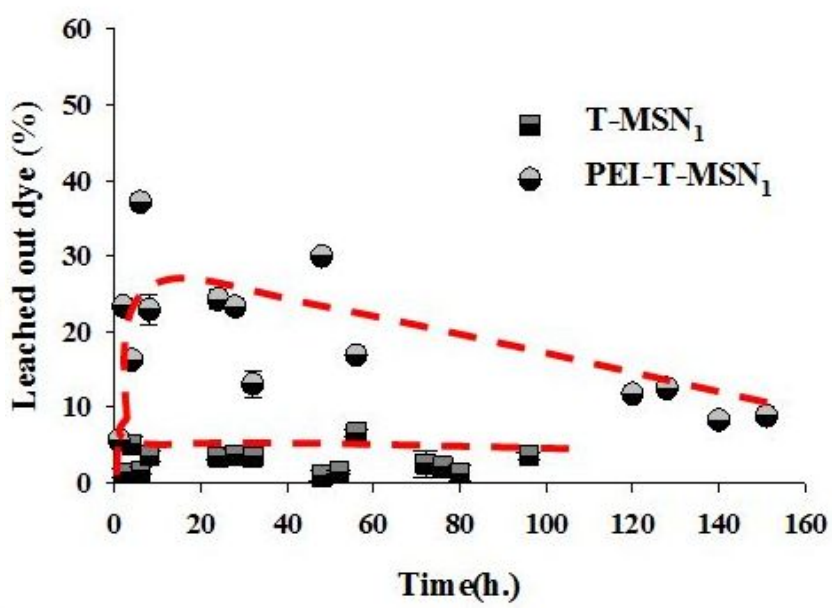

(b)

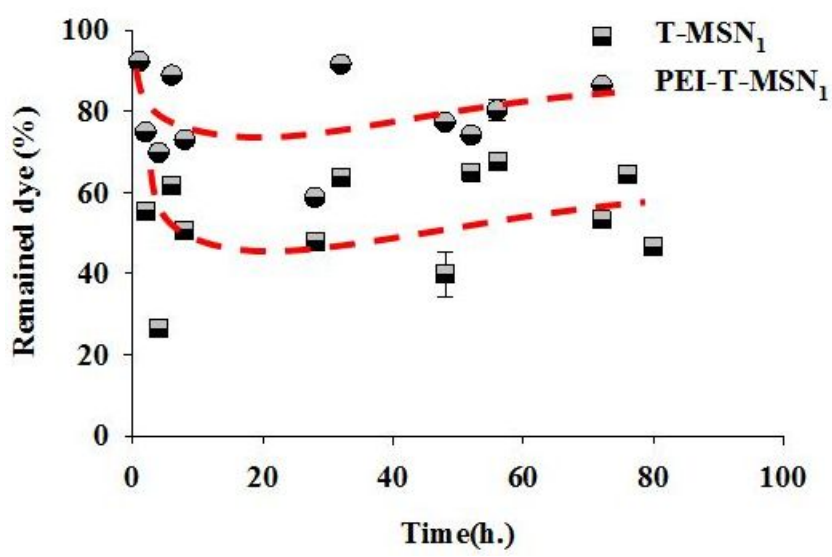

(d)

Fig. 7. Relative fluorophore leaching percentage based on measurement from the supernatant for F-MSN $\mathrm{N}_{1} \mathrm{~S}(\mathrm{a})$ and T-MSN $\mathrm{S}$ (b). Remaining fluorophore content in the leached MSNs, obtained by dissolution of the remaining MSN cake after separation from the leaching solution, for F-MSN 1 S (c) and T-MSN 1 S (d).

drodynamic size and surface charge measurements after the leaching experiments still yielded comparable results to those before the experiment (Supplementary Figure S4). Also the dye (re-)adsorption notation can be supported by these measurements, as all surface charges were comparable before and after leaching except in the case of T-MSN ${ }_{1}$. Here, the surface charge after leaching had shifted to positive from negative, indicating that an adsorption of positively charged species to the MSN surface must have occurred during the leaching experiment.

For the second analysis, based on separation of the remaining MSNs from the leaching media and dissolution in $\mathrm{NaOH}$ after drying with subsequent spectrophotometric determination, a similar trend was found for F-MSN ${ }_{1} \mathrm{~S}$ (Figure $7 \mathrm{a}, \mathrm{c}$ ), albeit for T-MSN $\mathrm{M}_{1} \mathrm{~s}$ the relationship was re- versed (Figure $7 \mathrm{~b}, \mathrm{~d}$ ). The $\mathrm{PEI}_{a d s}$-T-MSNs relative plateau value is still comparable between the two analysis methods, and the leaching degree is quite low as compared to what could be expected from the leaching behavior of F-MSN ${ }_{1} \mathrm{~S}$ if only the electrostatic interaction aspect is regarded, considering the $\mathrm{PEI}_{a d s}-\mathrm{T}-\mathrm{MSN}_{1} \mathrm{~s}$ and liberated rhodamine molecules should carry the same charge under the studied conditions. Possibly, the PEI-surface coating can actually function as a protective coating in this case, hindering the liberated dye molecules from diffusing out of the pores to a certain extent. The conformation of PEI under the studied conditions would be 'swollen' due to the high positive charge density, which could partly 'cap' or close the pores, as has been found for polyaminefunctionalized MSNs also in the past [40, 41]. 
Overall, we note that such degrees of fluorophore leaching would have substantial implications on the quantification of fluorophore-labeled MSNs under studied biological/ physiological conditions, and should thus carefully be taken into account when attempting to carry out such quantifications. Along with the inherent properties of the fluorophore itself, especially when it comes to the commonly applied FITC/Fluorescein, quantification based on fluorescence measurements becomes increasingly challenging. We also note that this is not exclusively a property of attached fluorophores, but any functional moiety that have been attached to the MSNs, such as chelates (for radiolabelling for nuclear imaging or Gd-complexation for MR-imaging) and/or targeting ligands. This aspect is further highlighted by the fact that the commonly utilized chelates, such as DOTA or DTPA, are often coupled via the same (or very similar) chemistry as the fluorescent dyes demonstrated here. The conditions applied here were static, but given 'unlimited' access to water (such as in the body) these effects may be even more pronounced, as for instance re-adsorption is less likely to occur. Other "uncounted for" effects that may either promote or suppress any of the abovementioned observations especially if the moiety is exposed on the particle surface, including presence of enzymes, adsorption of plasma proteins, influence of blood pressure (on dissolution and disintegration) may also come into play and should also thus be given room for consideration in the interpretation of results as well as already in the initial steps of the design process.

\subsection{Influence on photostability upon incorporation of fluorophores into MSNs}

For imaging purposes, the benefit in incorporation of a fluorescent dye into a MSN matrix could be assumed to be the same as for incorporated drugs, i.e. to provide a protective and stabilizing environment against the surrounding conditions [42]. In the specific case of fluorophores, the incorporation into such a ceramic matrix could intuitively lead to an enhanced photostability and quantum yield of the fluorophores [43]. These effects can be caused by the stabilizing, non-reactive matrix that prevents reactions with excited molecules and by disallowing dynamic quenching, besides providing a protective environment from the surroundings. The uniqueness in the MSN architecture as compared to other particle systems lies in its porous structure, where the fluorescent dye molecules can be homogeneously distributed and thus well-separated from each other, limiting the disadvantages associated with many other nanoparticle structures where the dyes are closely packed e.g. in the core of the particle. Such close-packing could consequently lead to quenching via some nonradiative pathway such as molecule-molecule interaction, electron transfer, or isomerization within the solid matrix [22]. Due to this reason, commercial fluorophores have previously been entrapped inside MSNs to produce "ultrabright fluorescent mesoporous silica nanoparticles" [4446]. In these cases, however, the dyes were only physically entrapped within the still surfactant-filled MSNs, making them unsuitable for bioprobe applications since the cationic $\mathrm{CTA}^{+}$templates used are highly toxic (see also section 3.8). However, enhanced photostability over free dye was asserted and, the relative brightness per particle was further claimed to be up to forty times of similarly sized quantum dots [46]. To investigate whether our surfactantfree, covalently conjugated fluorophores could also obtain any advantages in terms of photostability by incorporation into the MSN matrix, we measured the half-life of the MSNincorporated dyes based on number of imaging cycles needed to completely bleach the dye by laser-scanning confocal microscopy (LSCM) utilizing a laser intensity of approximately $8 \mu \mathrm{W} / \mu \mathrm{m}^{2}$. To investigate the photostability in a relevant setting from the application point of view, the measurements were conducted in a cellular environment. The samples consisted of equal fluorophore concentrations in terms of free dyes (FITC and TRITC) vs that conjugated to particles $\left(\mathrm{F}-\mathrm{MSN}_{1}, \mathrm{~T}-\mathrm{MSN}_{1}, \mathrm{PEI}_{\text {ads }}-\mathrm{F}-\mathrm{MSN}_{1}\right.$, $\mathrm{PEI}_{\text {ads }}$-T-MSN ${ }_{1}$ ). Thus, MSNs and corresponding amounts of free dyes were incubated with HeLa cells at a concentration of $10 \mu \mathrm{g} \mathrm{MSNs} / \mathrm{ml}$ for $6 \mathrm{~h}$, whereafter the cells were fixed. Nevertheless, as intracellular comparison of MSNs as compared to free dye was not possible due to the inability of the free hydrophilic dyes to penetrate the cell membrane by diffusion (as demonstrated in Figure 8), the comparison to free dyes were performed in cell lysate instead (Figure 9a,b).

To measure the photostability of MSN-conjugated fluorophores inside cells, regions of interest (ROI) were chosen inside endosomes i.e. where the particles were collected, and for each ROI the intensity decay due to photobleaching was measured by fitting a single exponential decay curve. For the FITC-MSNs (F-MSN ${ }_{1}$ and PEI Ids - F-MSN $_{1}$ ) the results were quite variable between measurements, so they have been omitted from the analysis. This may be related to the time point used, $6 \mathrm{~h}$ after incubation, whereby different fractions of free dye may already exist in the endosomes which complicates the analysis. For the TRITCMSNs (T-MSN 1 and $\mathrm{PEI}_{a d s}$-T-MSN ${ }_{1}$ ), however, a clear trend could be observed between the particles with and without $\mathrm{PEI}$, where the $\mathrm{PEI}_{a d s}-\mathrm{T}-\mathrm{MSN}_{1}$ s proved to be more photostable than the non-coated ones (Figure 9a). The contribu- 


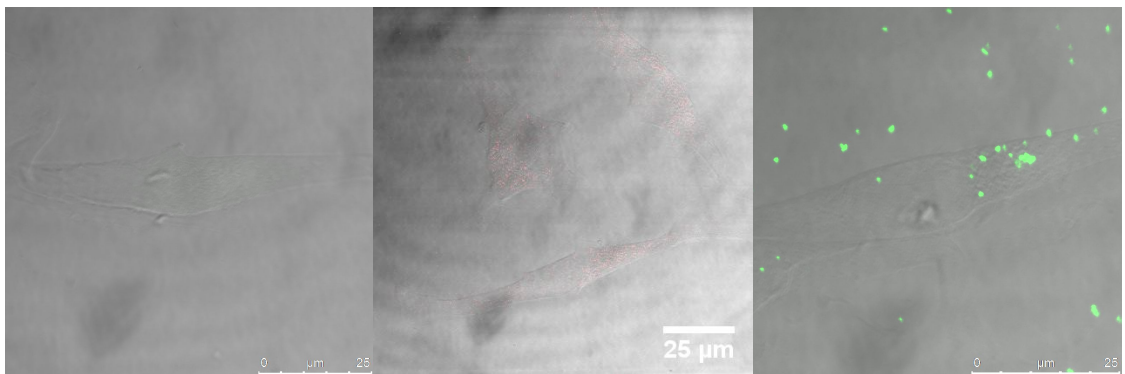

(a)

(b)

(c)

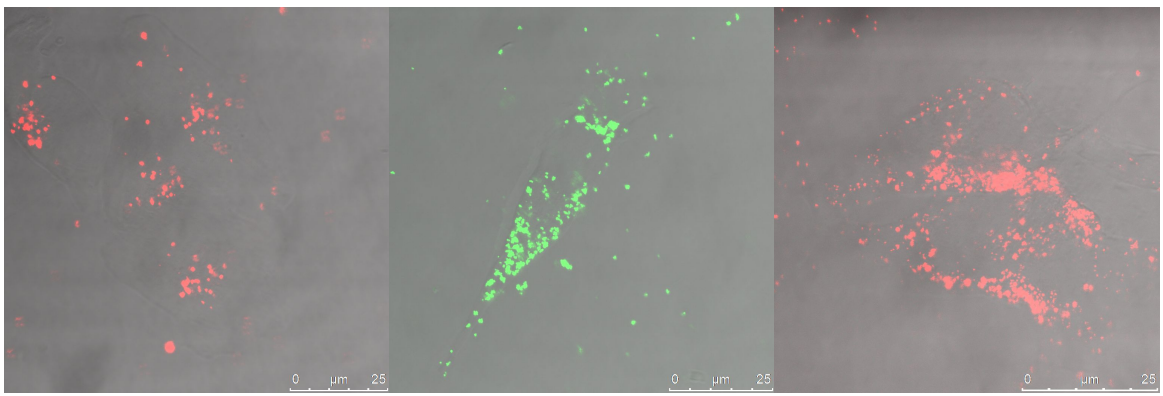

(d)

(e)

(f)

Fig. 8. Comparison of cellular internalization of free dyes vs MSN-immobilized dyes in fixed HeLa cells after $6 \mathrm{~h}$ of incubation. a) Free FITC dye, b) Free TRITC dye, c) F- $\mathrm{MSN}_{1} \mathrm{~s}$, d) T-MSN 1 s, e) PEI ${ }_{a d s}-\mathrm{F}-\mathrm{MSN}_{1} \mathrm{~s}$ and f) $\mathrm{PEI}_{a d s}-\mathrm{T}-\mathrm{MSN}_{1} \mathrm{~S}$.
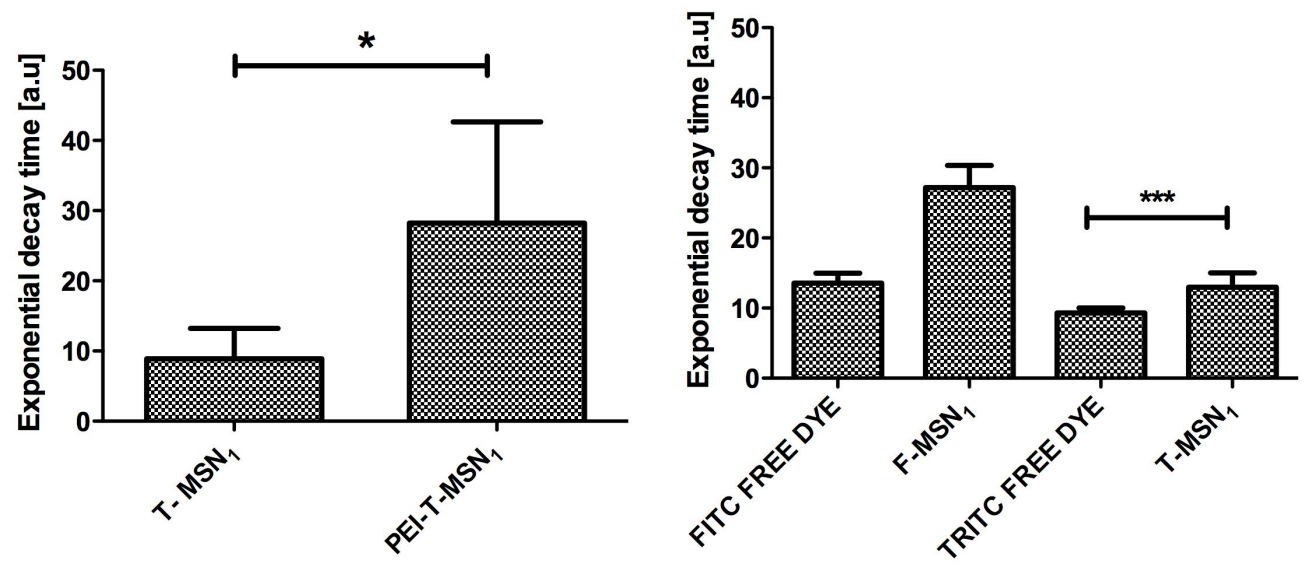

(a)

(b)

Fig. 9. Life-time measurements for MSN-tagged fluorophores in cellular environments. a) Comparison of fluorescence decay half-life of TRITC labeled $\mathrm{MSN}_{1}$ vs. PEl-adsorbed T-MSN 1 s in fixed HeLa cells. Data are expressed as mean \pm SD of six different experiments. ${ }^{\star} P \leq 0.05$. b) Comparison of fluorescence decay half-life of free fluorophores vs the same dyes when conjugated to MSNs in cell lysate. Equal amount of fluorophore concentration were used per sample.Data are expressed as mean \pm SD of three different experiments for group 1 (FITC free dye and F-MSN F $_{1}$ and 9 different experiments for group 2 (TRITC free dye and T-MSN ${ }_{1}$ ). ${ }^{\star \star *} \mathrm{P} \leq 0.001$.

tion of PEI as a protective coating thus seemed to be facilitative in this aspect also. In the lysate experiments (Figure 9b), it was clear that the MSN-conjugated dyes were more photostable than the free dyes, supporting the idea of the dyes sustaining enhanced photostability upon being incorporated into a solid matrix such as an MSN.

As a demonstration of applicability for the best candidate $\left(\mathrm{PEI}_{a d s}-\mathrm{T}-\mathrm{MSN}_{1}\right.$ ) from the above-presented results, 

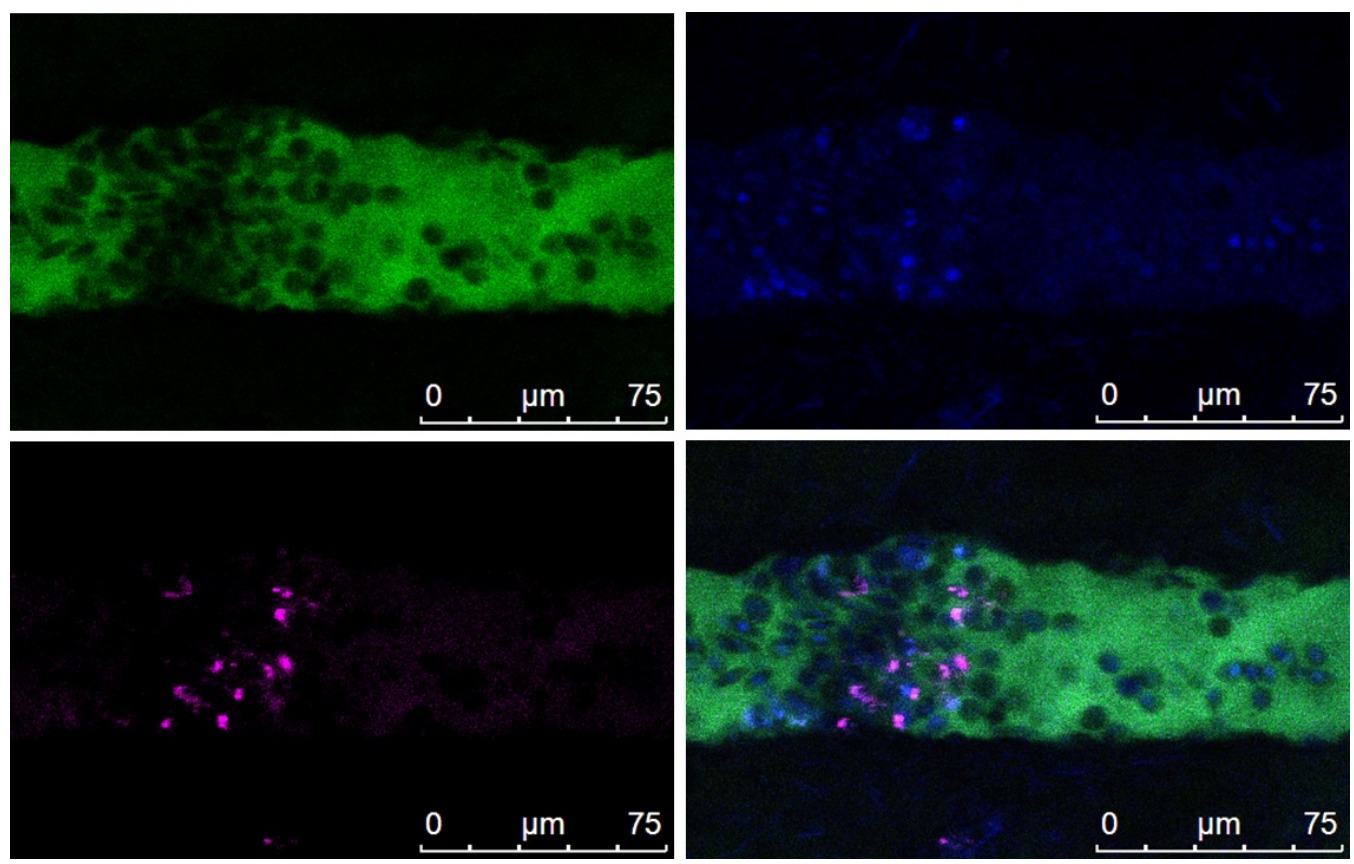

(a)

Fig. 10. Two photon in vivo imaging of PEG-PEI-T-MSN particles in CAM model. PEG-PEI-T-MSN ${ }_{1} \mathrm{~S}$ are detectable and fluorescently stable probes

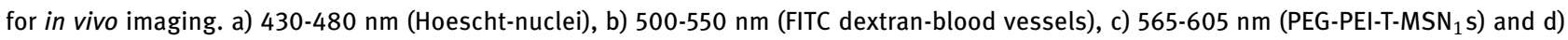
Overlay image.

and to verify that the obtained fluorescence upon MSN conjugation of rhodamine following this route allows detectability also in vivo, we performed live two-photon imaging of intravenously injected $\mathrm{PEI}_{a d s}-\mathrm{T}_{-} \mathrm{MSN}_{1} \mathrm{~s}$ in a chorioallatonic membrane (CAM) in vivo model. To possibly reduce any undesired effects upon administration via the intravenous route, $5 \mathrm{kDa}$ PEG chains were conjugated to the $25 \mathrm{kDa}$ PEI before coating via our previously reported procedures [47]. The circulating particles were clearly visible in the blood vessels (Figure 10) where the blood vessels were visualized by injection of a FITC-dextran conjugate and the blood cell nuclei were stained by a Hoechst dye 33342, confirming that fluorophore conjugation via the standard routes for MSNs (co-condensation via aminopropylsilanes) employed here was indeed sufficient also for reaching in vivo detectability in the described embodiment.

\subsection{Influence of surface coating on cellular uptake and viability}

Having established that surface functionalization may have pronounced implications on the fluorescence properties of MSNs, evaluation of the influence of different surface coatings on some of the most basic aspects of the MSN biobehavior was undertaken. In our previous study on the influence of particle shape (rods vs spheres) on cellular uptake, we also observed some distinct surface chargedependent effects [14]. In the present case, to isolate the surface coating effects from the shape-induced differences, a similar series of particles (denoted $\mathrm{MSN}_{2}$ ) was prepared, including PEG-functionalized MSNs that was not included in our former study. $5 \mathrm{kDa}$ PEG was conjugated either directly to the MSN surface (PEG-MSN 2 ) via the cocondensed amino groups, or as mediated by the surfacegrown hyperbranched PEI layer (PEG-PEI ${ }_{\text {graft }}-\mathrm{MSN}_{2}$ ). The PEI layer was also derivatized with succinic acid groups (Succ-PEI $\mathrm{Praft}_{\text {- }}-\mathrm{MSN}_{2}$ ) and the same treatment regime was carried out for the non-coated MSN particle (Succ-MSN ${ }_{2}$ ). This yielded particles with negative surface charge at neutral $\mathrm{pH}$, where the effect was more pronounced for Succ$\mathrm{PEI}_{\text {graft }}$-MSNs due to the high surface density of modifiable functional groups provided by the PEI layer (Table 1). Upon PEGylation, the charge was only reduced by increasing the electrical double layer thickness, whereby the zeta potential is determined further away from the actual particle surface; whereas the PEG chains do not contribute to the charging themselves since they do not contain any chargeable groups. The plain MSN particle, for which 10\% aminosilane was used as co-silica source in order to have a high enough amount of modifiable groups, had a close- 
to neutral but slightly positive charge. The characteristics of the differently functionalized particles in aqueous suspension were summarized above in (Table 1). Since every small modification of a nanoparticle may have an impact on the biobehavior of the particle, cell viability for the whole series was carried out at two different concentrations on HeLa cancer cells (Supplementary Figure S5). None of the particles exhibited any significant effect on the cell viability at the studied concentrations.

Having established that the MSN series was safe to use for further cellular studies, the influence of surface modification on cellular uptake was studied on two different cancer cell lines of different origin, using both fluorescenceassisted cell sorting (FACS) and fluorescence confocal microscopy. The employed cell lines were HeLa or Human cervical adenocarcinoma cells, which is the mostly studied human cancer cell line with a fibroblastic morphology; and Caco-2 or Human colon adenocarcinoma cells, which have the ability to differentiate and polarize under specific conditions, and thus is a morphological and functional model for the enterocyte lining of the small intestine. The Caco- 2 cell line and low particle concentrations $(5 \mu \mathrm{g} / \mathrm{ml})$ were chosen to be able to better discern differences between the differently functionalized MSNs [14].

For the HeLa cells, a high surface charge, positive or negative, seemed to be beneficial for cellular uptake. From the Caco- 2 cellular uptake can be deduced that the most preferential coating is, in fact, the PEG-PEI coating; despite a lower net charge. Even though the cellular uptake is certainly cell-line dependent also, the trends observed for the two cell lines used here is quite similar (Figure 11). In the case of the PEG-PEI coating, the addition of PEG cannot be the single decisive component since conjugation of PEG directly to the MSN surface yielded the lowest uptake $\%$ for both cell lines, i.e. a reduction as compared to its "starting particle"; whereas conjugation of PEG to the $\mathrm{PEI}_{\text {graft }}{ }^{-}$ $\mathrm{MSN}_{2}$ lead to an increased uptake in the case of Caco-2. Comparable results were obtained by analysis of the mean fluorescence intensity (MFI) values (Supplementary Figure S6) but given the uncertainties around quantification connected to the fluorescent label used (fluorescein) discussed above, we rather base our analysis on \%positive cells. The same trends seems to hold true for HeLa cells, especially when examining the confocal microscopy images (Figure 12), where slightly more green MSN signals can be observed within the cells incubated with PEG-PEI graf $^{-}$ $\mathrm{MSN}_{2} \mathrm{~S}$ (Figure 12d) than $\mathrm{PEI}_{\text {graf }}-\mathrm{MSNs}_{2}$ (Figure 12b). Based on microscopy analysis, these two coatings were clearly superior as compared to the other ones in providing an efficient cellular internalization. We note that despite an adequate uptake for the succinylated particles in FACS, these particles were harder to visualize (Figure 12e,f) due to the relatively lower fluorescence intensity of these particles (Table 1). To pinpoint the differences in cellular uptake, which are clearly not based on surface charge alone, a deeper look into the involved mechanisms and the effect of the surface coating were attempted below.

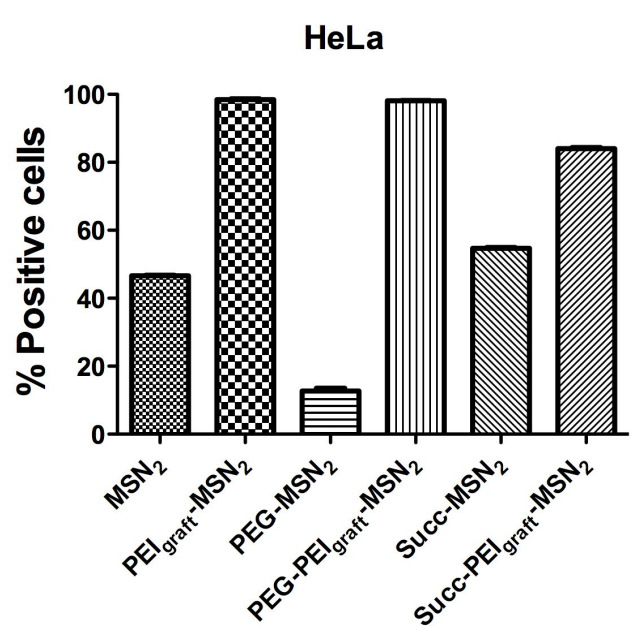

Type of Particle

(a)

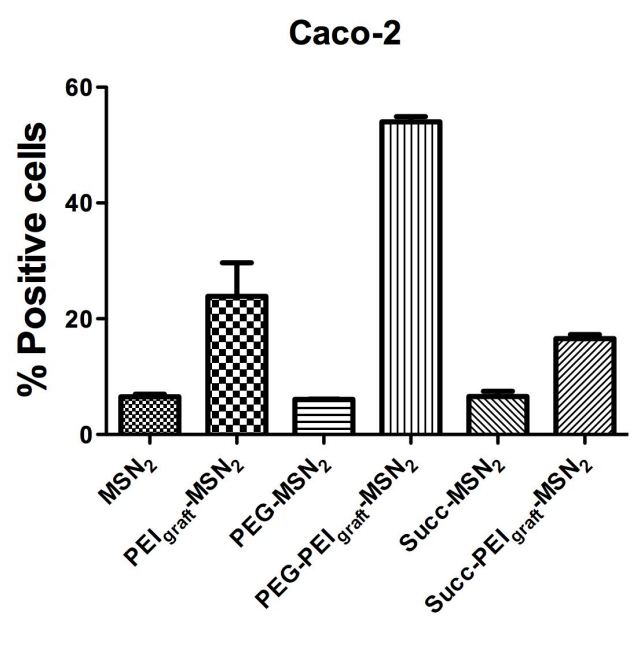

Type of Particle

(b)

Fig. 11. Effect of surface functionalization on MSN uptake in two different cancer cell lines. Flow cytometric investigation of $\mathrm{MSN}_{2} \mathrm{~S}$ ( $5 \mu \mathrm{g} / \mathrm{mL}$ ) uptake in a) HeLa and b) Caco-2 cells after $4 \mathrm{~h}$ incubation. Data are expressed as mean $\pm \mathrm{SEM}$ of three independent experiments. 

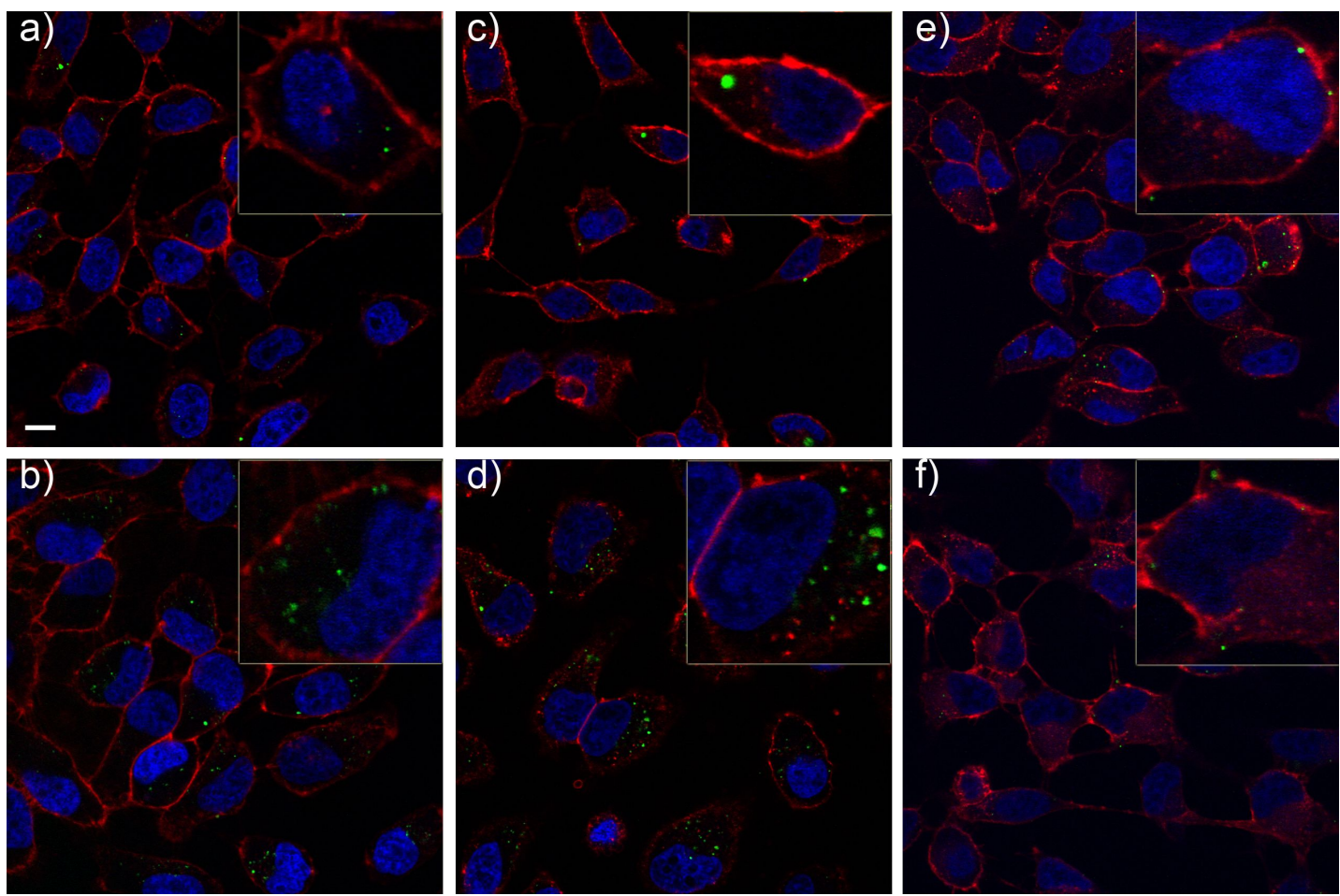

Fig. 12. Confocal microscopy images of HeLa cells incubated with a) $\mathrm{MSN}_{2}$, b) $P \mathrm{PL}_{\text {graft }}-\mathrm{MSN}_{2}$, c) PEG-MSN 2 , d) PEG-PEI graft $^{-M S N_{2}}$, e) Succ$\mathrm{PEI}_{\text {graft }}-\mathrm{MSN}_{2}$ particles, $5 \mu \mathrm{g} / \mathrm{mL}$ (green) for $4 \mathrm{~h}$. Cell nucleus stained using DAPI (blue) and cell membrane stained using rhodamine-lectin (red) (Inset showing zoomed image of the internalized MSNs).

\subsection{Influence of surface charge on route of uptake}

Nanoparticles are generally taken up by cells via endocytosis, which is a specialized cell transport mechanism to internalize small molecules, macromolecules and particles. Generally, endocytosis can be classified into phagocytosis and non-phagocytic endocytosis. Phagocytosis involves the uptake of large particles and is one of the main mechanisms by which particles are taken up by macrophages and neutrophils. Non-phagocytic endocytosis takes place either through macropinocytosis, clathrincoated pits, through caveolae, or through clathrin and caveolae independent pathways. The different endocytotic processes vary in their vesicle size, proteins involved in the vesicle formation, and the cell type in which they are found. After internalization, the intracellular fate is dependent on the endocytosis pathway. The endocytotic internalization and appropriate intracellular trafficking is thus a perquisite for effective performance of drug/gene carriers [48]. Macropinocytosis is a bulk internalization process, where the intracellular fate of macropinosomes varies depending on the cell type, but in most cases, they acidify and shrink. They may eventually fuse with lysosomal compartments or recycle their content to the surface. Clathrin-mediated endocytosis, can be receptordependent or independent, causes the endocytosed material to end up in the lysosome via "early" endosomes [49], whereas caveolae-mediated endocytosis is a highly regulated process, involved in endocytosis and trancytosis of various proteins. The advantage of caveolae-mediated endocytosis is to by-pass lysosomal degradation. Ligands for caveolae-mediated endocytosis include folic acid, cholesterol and albumin.

For MSNs, the primary route of uptake has been found to be of clathrin-mediated type [13] except in the case of highly positively charged particles, whereby the uptake route may be of undefined (other/unknown endocytic pathway) type, as it has been shown that it could not be inhibited by any endocytic inhibitor [50]. Size-dependency experiments have shown that Polystyrene (PS) spheres of size $100 \sim 200 \mathrm{~nm}$ are preferentially internalized via the clathrin-mediated route, whereas PS spheres of $500 \mathrm{~nm}$ would be taken up primarily via the caveoale-mediated endocytosis route. Since our studied MSNs were, in this regard, of intermediate size $(\sim 300 \mathrm{~nm})$ and represented both extremes of the surface charge spectrum - highly positive and highly negative, as well as close to neutral - we set out to investigate via which route of uptake these MSNs of different characteristics would be internalized by ap- 
plying different uptake inhibitors. Again, to better be able to discern differences, Caco-2 cells were used instead of HeLa [14]. To determine whether MSN uptake was an active or passive process, cells were energy depleted using sodium azide $\left(\mathrm{NaN}_{3}\right)$, which is known to inhibit the respiratory chain in the mitochondria, thus impairing the production of ATP in the cell and consequently the active uptake. Macropinocytosis can be inhibited by amiloride, which is a selective inhibitor of $\mathrm{Na}^{+} / \mathrm{K}^{+}$exchange, blocking macropinocytosis by lowering submembraneous $\mathrm{pH}$ (cytosolic $\mathrm{pH}$ close to the membrane). Clathrin-mediated endocytosis can be inhibited by potassium depletion, or by inhibitors such as phenyl arsine oxide (PAO) and chlorpromazine. PAO, a trivalent arsenical, reacts with vicinal sulfhydryls to form a stable ring-structure and inhibits clathrin-mediated endocytosis by crosslinking the clathrin coat. Inhibitors of caveolae-mediated endocytosis include filipin, nystatin, methyl- $\beta$-cyclodextrin and genistein. Genistein, is a tyrosine kinase inhibitor, inhibits caveolae- mediated endocytosis.

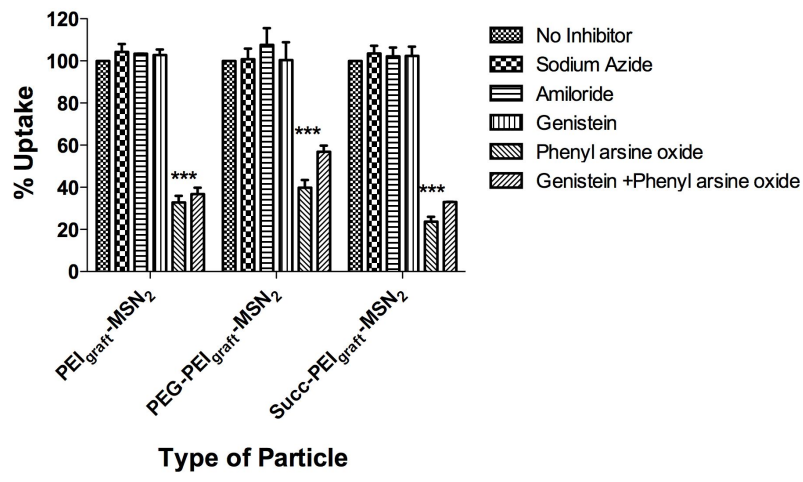

Fig. 13. The effects of various uptake inhibitors on MSN uptake in Caco- 2 cells.Cellular uptake of differently charged $\mathrm{MSN}_{2} \mathrm{~S}$ after cotreatment with Sodium azide, Amiloride, Genistein, Phenyl arsine oxide and Genistein + Phenyl arsine oxide. Data are expressed as mean \pm SEM of three to four independent experiments. ${ }^{*} P \leq 0.05$, ${ }^{*} P \leq 0.01$ and ${ }^{* *} P \leq 0.001$ as compared with respective MSN control without inhibitor.

As can be seen in Figure 13, the uptake of all $\mathrm{MSN}_{2} \mathrm{~S}$, regardless of surface functionality, were inhibited by the addition of phenyl arsine oxide as well as, to a similar degree, by genistein in combination with PAO. This signifies that all the $\mathrm{MSN}_{2} \mathrm{~S}$ were mainly internalized by the clathrinmediated route regardless of surface charge. Whereas inhibition by the combination of PAO and genistein was not further inhibiting the uptake, but it was actually slightly increasing the total uptake, this suggests that there might

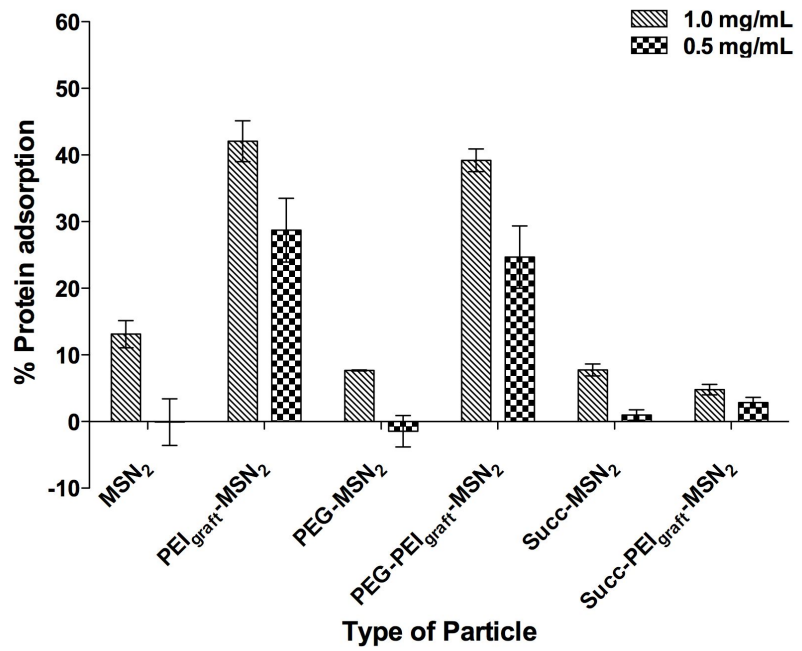

Fig. 14. Relative serum protein adsorption on mesoporous silica nanoparticles at two different $\mathrm{MSN}_{2}$ concentrations.

be some other/clathrin- and caveolae-independent pathway, which can be activated by inhibition of both the pathways. Thus, our surface-charge independent results are in contradiction with earlier results [51] that suggested that high positive charge would induce a different route of uptake. One explanation for this observation may be the extent of serum protein adsorption on our MSNs of different surface functionality, as the adsorbed proteins are also charged species and would hence contribute to the overall surface charge. All our cellular experiments have been conducted in the presence of serum, whereas in the aforementioned reference the route of uptake was determined in the absence of serum [51]. The presence or absence of serum is known to affect the route of uptake, whereby the clatrhin-mediated route has been found to be the preferred route of uptake for particles that has adsorbed serum proteins on their surfaces $[52,53]$.

\subsection{Influence of surface coating on serum protein adsorption}

While the extent of serum protein adsorption from the cell media thus could affect the route of uptake under in vitro conditions, the main rationale for PEGylation of nanomaterials is its expected property in diminishing protein adsorption in vivo [54, 55]. This highly sought-for trait is commonly referred to as 'stealth' properties, which directly implicates that reducing the amount of plasma protein adsorption on the particle surface, also reduces the likelihood of the body defense mechanisms (reticuloendothe- 

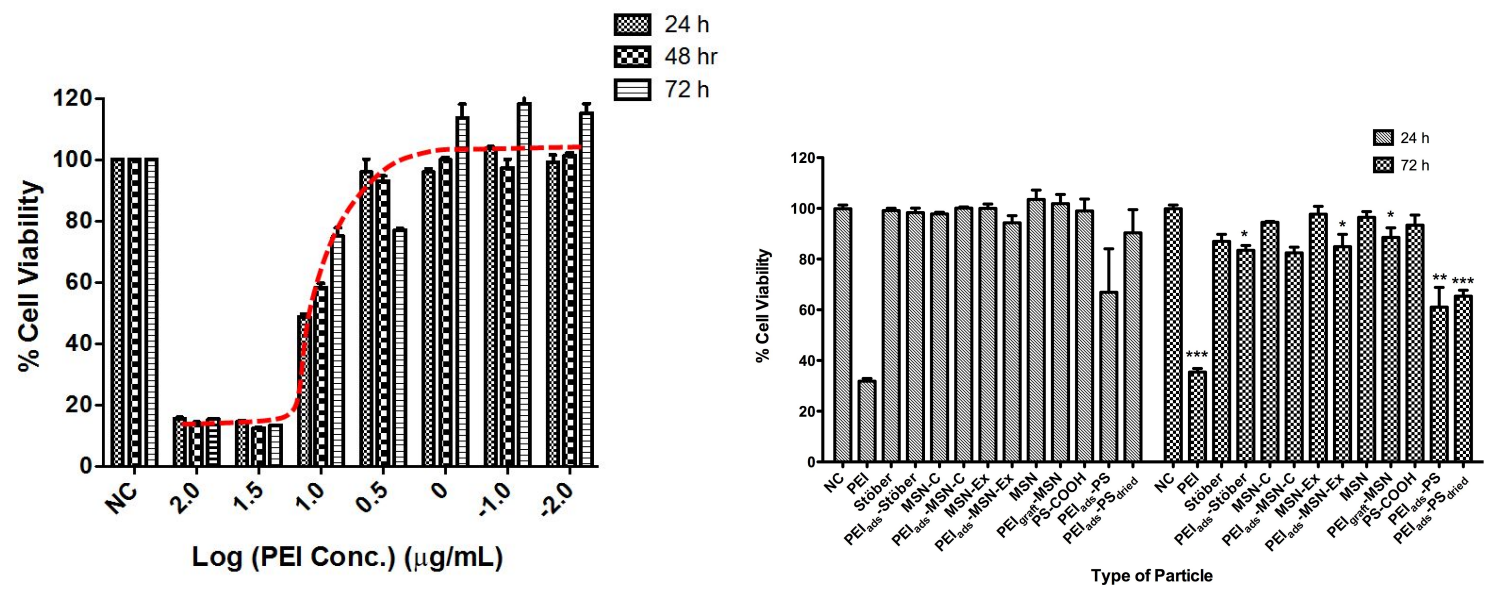

(a)

(b)

Fig. 15. Cell viability of HeLa cells incubated with a) different concentrations of PEI ( $25 \mathrm{kD})$ for $24 \mathrm{~h}, 48 \mathrm{~h}$ and $72 \mathrm{~h}$ b) different particles with and without coated PEI equivalent to $10 \mu \mathrm{g} / \mathrm{mL}$ PEI (25 kD) for 24 and $72 \mathrm{~h}$, evaluated by the WST-1 assay. Polystyrene (PS) particles of similar size were included as particle control. Data are expressed as mean \pm SEM of three to four independent experiments.

lial system, RES/mononuclear phagocyte system, MPS) of recognizing the particles as foreign. Uptake by the RES/MPS would, in turn, result in rapid elimination of the circulating particles from the blood stream. Achieving 'stealth' properties would consequently prolong the circulation time in the blood, which increases the likelihood of the particles reaching their target. Long-circulating properties are thus one of the main goals associated with injectable nanoformulations, especially for targeted drug delivery. If active targeting is pursued, the adsorbed proteins, a.k.a. the "protein corona", should also not cover up the targeting ligand ("biofouling") and consequently hamper target recognition. The reasons for PEG being the leading stealth coating candidate is connected to its hydrophilicity (where hydrophobic particles are known to rapidly be covered with plasma proteins) and neutral charge (high charge, either positive or negative, are believed to promote protein adsorption). Besides PEGylation, zwitterionic surface coatings have been suggested to provide similar 'stealth' properties $[12,56]$. In this sense, to determine whether the same holds true for our MSNs, serum protein adsorption was conducted at two different concentrations in HEPES buffer ( $\mathrm{pH}$ 7.2) for the whole $\mathrm{MSN}_{2}$ series.

The difference in ability to suppress adsorbed serum proteins between surface coatings was quite distinct (Figure 14). Large amounts of serum proteins was adsorbed on the PEI-MSN ${ }_{2} \mathrm{~S}$, probably associated with its high surface charge along with the fact that it is positive, since the majority of serum proteins are negatively charged. Further, the PEGylation of this particle (PEG-PEI graft $-\mathrm{MSN}_{2}$ ) lead to very slight reduction in protein adsorption; and the ef-
Table 2. Zeta potential measurements of MSN particle suspensions in HEPES before and after incubation in DMEM cell media containing $10 \%$ serum.

\begin{tabular}{lcc}
\hline Type of Particle & $\begin{array}{c}\text { in HEPES } \\
(200 \mu \mathrm{g} / \mathrm{mL})\end{array}$ & $\begin{array}{c}\text { after } 2 \mathrm{~h} \text { incubation in cell } \\
\text { media redispersed in HEPES } \\
(200 \mu \mathrm{g} / \mathrm{mL})\end{array}$ \\
\hline \hline $\mathrm{MSN}_{2}$ & +5.4 & -14.8 \\
PEI $_{\text {graft }}-\mathrm{MSN}_{2}$ & +47.9 & +2.7 \\
PEG-MSN $_{2}$ & +0.2 & -5.8 \\
PEG-PEI $_{\text {graft }}-\mathrm{MSN}_{2}$ & +15.2 & +9.1 \\
Succ-MSN $_{2}$ & -14.4 & -17.0 \\
Succ-PEI $_{\text {graft-MSN }}$ & -61.6 & -37.1 \\
\hline
\end{tabular}

fect of PEGylation of the pure $\mathrm{MSN}_{2}$ was also not drastic but discernible. For the pure $\mathrm{MSN}_{2}$, the succinylation effect was also not that drastic; whereas succinylation of the $\mathrm{PEI}_{\text {graft }}-\mathrm{MSN}_{2}$ lead to the lowest observed serum protein adsorption overall. Given that the absolute charge value of this particle was the highest of the whole series $(\sim 60 \mathrm{mV})$, this stands in sharp contrast to the notation that high surface charge promotes protein adsorption. However, given the zwitterionic nature of this coating, this observation does indeed stand in line with previous claims [12, 56]. Thus, for our MSN system, employing the zwitterionic approach was drastically more efficient than PEGylation in diminishing serum protein adsorption under the studied conditions.

In order to try to combine the above observations with those of cellular uptake from a surface charge point of view, the net surface charges of the MSNs were investi- 
Table 3. Physico-chemical characterization of particles used for PEI toxicity tests.

\begin{tabular}{|c|c|c|c|c|}
\hline Type of Particle & Hydrodynamic diameter (nm) & $\zeta$-potential $[\mathrm{mV}] \mathrm{pH}=7.2$ & $\%$ w/w PEI loading & conc. used $(\mu \mathrm{g} / \mathrm{mL})$ \\
\hline Stöber & 170 & -44.9 & - & 555.6 \\
\hline $\mathrm{PEI}_{\mathrm{ads}}$-Stöber & 205 & +47.4 & 1.8 & 555.6 \\
\hline MSN-C & 353 & -40.0 & - & 142.8 \\
\hline $\mathrm{PEI}_{\text {ads }}-\mathrm{MSN}-\mathrm{C}$ & 248 & +46.9 & 7.0 & 142.8 \\
\hline MSN-Ex & 871 & -26.7 & - & 67.6 \\
\hline $\mathrm{PEI}_{\mathrm{ads}}-\mathrm{MSN}-\mathrm{Ex}$ & 224 & +44.4 & 14.8 & 67.6 \\
\hline MSN & 248 & +5.3 & - & 58.1 \\
\hline PEI $_{\text {graft }}-M S N$ & 291 & +55.9 & 17.2 & 58.1 \\
\hline PS-COOH & 153 & -56.2 & - & 500.0 \\
\hline $\mathrm{PEI}_{\mathrm{ads}}-\mathrm{PS}$ & 207 & +44.1 & 2.0 & 500.0 \\
\hline
\end{tabular}

gated after $2 \mathrm{~h}$ incubation in DMEM cell media containing $10 \%$ serum protein (Table 2) afterwhich they were separated by centrifugation and redispersed in HEPES buffer. For all studied particles, the net surface charge decreased to more negative, except for Succ-PEI ${ }_{\text {graft- }} \mathrm{MSN}_{2}$. This suggests that the small portion of proteins adsorbed to these particles may, for the majority fraction, even be positively charged. For the other particles, the extent of protein adsorption reflects the decrease in zeta potential quite well, except for the PEG-PEI graft $_{-} \mathrm{MSN}_{2}$, for which a high protein adsorption is observed but, the reduction in zeta potential is only a few $\mathrm{mV}$. A PEGylated surface may certainly attract different proteins as compared to the pure PEI surface, which makes the resulting charge challenging to predict, since no common 'rule' for such protein selection on adsorption to surfaces exists to date [57]. Based on these results, the superior cellular uptake (Figure 11) resulting from these two coatings (PEI and PEG-PEI) could be due to the overall retained positive charge, which should indeed be facilitative from a cellular uptake point of view $[13,26$, 27].

\subsection{Influence of PEI coating on cytotoxicity}

Based on all above-presented results, PEI seems to be an especially useful MSN functionalization platform. However, there are certain concerns related to the usage of PEI in drug delivery context, since especially commercial $25 \mathrm{kDa}$ PEI has been found to be quite cytotoxic by itself $[58,59]$. Still, it remains the one of the most efficient non-viral gene delivery agents, but this kind of polyplex system (PEI-condensed DNA) will not be applicable in vivo (partly) due to this reason. Besides this study, we have utilized PEI as a basis for further MSN modification in many of our previous studies, due to the advantages mentioned previously (section 3.1). Specifically from a surface functionalization point of view this is a highly viable platform, due to its high abundance of reactive primary amino groups that can be utilized for very efficient further modification. So far, we have not observed any adverse effects, either in vitro $[14,16]$ or in vivo [60] utilizing our $\mathrm{PEI}_{\text {graft }}$ - $^{-}$ MSN platform. On one hand, we have intuitively ascribed this to the structure of this PEI coating, where surface hyperbranching polymerization is applied, and thus results in individual hyperbranched groups grown from the particle surface. In the investigation of such growth fashion of PEI on planar surfaces, it has been found that the growth usually stops after 3-4 generations due to steric encumbrance [61]. Thus, the molecular weight of the individual branches should not be very high. In this respect, Zink and co-workers performed a study where they adsorbed commercial PEI of different molecular weights $(0.6,1.2,1.8$, 10 and $25 \mathrm{kDa}$ ) on MSNs and investigated their cytotoxicity [62]. They found that only the high-molecular weight $(25 \mathrm{kDa})$ PEI could induce cytotoxicity in this case, with slight induction of cytotoxicity of the $10 \mathrm{kDa} \mathrm{PEI}$ on one of the cell lines used (PANC-1). They thus ascribed the PEIrelated toxicity to be connected to its molecular weight. On the other hand, we have tentatively assumed a contribution on lessening the toxic effect related to the extremely high positive charge density of PEI as being partly 'neutralized' to some degree by dissolving silica. Such 'biodegradation' of the material itself (MSN) could create a concentration gradient of silicic acid, which could thus possibly counteract the PEI effect. To verify our assumptions, we first determined the dose-response curve of $25 \mathrm{kDa}$ PEI toxicity on HeLa cells (Figure 15a). Subsequently, we adsorbed the same kind of PEI onto three different silica particles, non-porous Stöber, solvent-extracted MSNs and calcined MSNs; as well as a polystyrene (PS) particle of comparable (resulting) size via electrostatic interactions. The particles were separated, washed, dried, afterwhich the PEI amount was determined by TGA (Table 3). An MSN sample with cor- 
responding amount of surface-grafted PEI ( $\left.\mathrm{PEI}_{\text {graft }}-\mathrm{MSN}\right)$ as that of the solvent-extracted adsorbed PEI ( $\mathrm{PEI}_{a d s}-\mathrm{MSN}$ Ex) was included. We then chose a PEI concentration in the toxic range, in the present case $10 \mu \mathrm{g} / \mathrm{ml}$ pure $\mathrm{PEI}$, and incubated cells with the amount of each particle that was coated with the equal amount of PEI (Table 3) as well as the corresponding amount of plain particles without PEI, to distinguish the PEI-induced toxicity. These particles were incubated with HeLa cells for 24 and $72 \mathrm{~h}$ whereby viability was determined.

Clearly, all particle formulations were less toxic than PEI alone, despite the immensely high particle concentrations used ( $555 \mu \mathrm{g} / \mathrm{ml}$ for the highest concentration). For the PEI-PS particle, the toxicity was higher than for the PEI-MSNs, which could further also be attributed specifically to PEI-induced toxicity, as the plain PS particle did not induce considerable toxicity. None of the silica or silica-PEI combinations exhibited any detectable cytotoxicity at $24 \mathrm{~h}$, however at $72 \mathrm{~h}$ some slight signs of toxicity could be discerned. Interestingly, the level of toxicity detected did not correlate with particle concentration, but rather with the $\mathrm{PEI} / \mathrm{SiO}_{2}$ ratio. The viability for the PEIMSN-Ex was clearly the lowest among the silica-based particles; and for the same sample, the $\mathrm{PEI} / \mathrm{SiO}_{2}$ ratio was also the highest $(\sim 15 \mathrm{wt} \%)$. The viability levels for PEI-MSN$\mathrm{C}$ and PEI-Stöber were comparable; however, the inherent toxicity induced by the Stöber particles alone (without PEI) was higher than that of MSN-C, probably due to the much higher particle concentration used. For the pristine MSNs, there can also be a slight reduction in viability deduced for the MSN-Ex as compared to the MSN-C, despite a higher concentration used for MSN-C. We attribute this effect to the notion that solvent-extracted MSNs have also previously been found to be more toxic than their calcined counterparts, due to residual surfactant residing in MSNs when only subject to solvent extraction [63]. In the case of surface-grafted PEI, the results were comparable to that of the corresponding sample with electrostatically adsorbed PEI. Interestingly, the $\mathrm{PEI} / \mathrm{SiO}_{2}$ ratios were also similar for these samples, further stressing the idea that it is the $\mathrm{PEI} / \mathrm{SiO}_{2}$ ratio that is decisive rather than PEI amount. In this case, we do not see a molecular weight-induced effect, assuming that the grafted individual PEI functions are smaller than the commercial $25 \mathrm{kDa}$ one. In conclusion, the difference between (soluble/degradable) silica and (insoluble/inert) PS seems distinguishable. Based on this experiment alone we cannot attribute the difference between $\mathrm{SiO}_{2}$ and $\mathrm{PS}$ to dissolution/degradation behavior only; but we can infer that the well-known toxicity of PEI can, in fact, be circumvented/repressed when used as part of a composite material, especially together with silica.

\section{Conclusions}

In this study, we have highlighted some important aspects to be considered in the design of MSNs for biomedical applications with the aid of illustrative demonstrations. For the particles to be traceable in a biological/physiological environment, MSNs are typically tagged with fluorescent dyes. On one hand, when attempting to quantify particle uptake/biodistribution based on dye-labeled MSNs it should be kept in mind that the inherent properties of the dye itself, surrounding conditions, surface coating, and silica dissolution consequently leading to leaching of attached labels may have a tremendous impact on the quantification. On the other hand, the incorporation of the dye into the MSN matrix and further coating of the MSNs may even have beneficiary influences on the dye in terms of photostability enhancement, providing a protective environment and elimination of $\mathrm{pH}$-induced effects. In the present case, we also took advantage of the $\mathrm{pH}$-sensitivity of the common dye fluorescein to probe the local $\mathrm{pH}$ inside the pores of surface-functionalized MSNs. Surface functionalization, in turn, had a distinct impact on the magnitude of cellular uptake and degree of serum protein adsorption, whereas the route of uptake or toxicity did not seem to be affected by the nature or charge of the surface coating of our MSNs. Special consideration was devoted to PEI coatings, both in adsorbed and surface hyperbranched form, as it provides an especially useful platform for further functionalization due to its high abundance of reactive terminal amine groups. The characteristic properties of PEI were influential on the MSNs also, in that it could function as a "proton trap" keeping the local pH acidic due to its high buffering capacity. Derivatization of PEI with carboxylic acid groups lead to a zwitterionic surface coating with superior 'stealth' properties as compared to PEGylation in our case. The PEG-PEI combination, nevertheless, provided the highest cellular uptake and homogeneous intracellular distribution, comparable to that of the PEI coating alone. Given the versatility of the PEI coating, regardless of the coating procedure (surface grafting/electrostatic adsorption), we finally determined that the well-known cytotoxicity of large-molecular weight PEI could be mitigated when used as a component in a hybrid material together with silica, even at very high particle concentrations (up to 100x of that used in our cellular studies). The general trends presented here in an MSN context should thus be especially useful in judging the feasibility of different MSN designs for biomedical applicability. 
Acknowledgement: The 'Medicinska Understödsföreningen Liv och Hälsa' and 'Tor, Joe och Pentti Borgs Minnesfond' foundations (D.D.), Center for Functional Materials (D.S.K.), Graduate School for Materials Research (A.D.) as well as the Academy of Finland (project decisions \#137101, \#140193, \#260599) are greatly acknowledged for financial support.

\section{References}

[1] Jessica M Rosenholm et al., "Nanoparticles in Targeted Cancer Therapy: Mesoporous Silica Nanoparticles Entering Preclinical Development Stage," Nanomedicine 7, no. 1 (January 2012): 111-120, doi:10.2217/nnm.11.166.

[2] Yu-Shen Lin, Katie R. Hurley, and Christy L. Haynes, "Critical Considerations in the Biomedical Use of Mesoporous Silica Nanoparticles," The Journal of Physical Chemistry Letters 3, no. 3 (February 2, 2012): 364-374, doi:10.1021/jz2013837.

[3] Yanes RE, Tamanoi F. Development of mesoporous silica nanomaterials as a vehicle for anticancer drug delivery. Ther Deliv. 2012 Mar;3(3):389-404. doi: 10.4155/tde.12.9.

[4] Zongxi Li et al., "Mesoporous Silica Nanoparticles in Biomedical Applications," Chemical Society Reviews 41, no. 7 (March 12, 2012): 2590-2605, doi:10.1039/C1CS15246G.

[5] Piaoping Yang, Shili Gai, and Jun Lin, "Functionalized Mesoporous Silica Materials for Controlled Drug Delivery," Chemical Society Reviews 41, no. 9 (April 9, 2012): 3679-3698, doi:10.1039/C2CS15308D.

[6] Mohammad-Ali Shahbazi, Barbara Herranz, and Helder A. Santos, "Nanostructured Porous Si-Based Nanoparticles for Targeted Drug Delivery," Biomatter 2, no. 4 (October 1, 2012): 296312, doi:10.4161/biom.22347.

[7] Wilson X. Mai and Huan Meng, "Mesoporous Silica Nanoparticles: A Multifunctional Nano Therapeutic System," Integrative Biology 5, no. 1 (December 17, 2012): 19-28, doi:10.1039/C2IB20137B.

[8] Derrick Tarn et al., "Mesoporous Silica Nanoparticle Nanocarriers: Biofunctionality and Biocompatibility," Accounts of Chemical Research 46, no. 3 (March 19, 2013): 792-801, doi:10.1021/ar3000986.

[9] Qianjun He and Jianlin Shi, “MSN Anti-Cancer Nanomedicines: Chemotherapy Enhancement, Overcoming of Drug Resistance, and Metastasis Inhibition," Advanced Materials (2013): n/an/a, doi:10.1002/adma.201303123.

[10] Yu Chen, Hangrong Chen, and Jianlin Shi, "In Vivo BioSafety Evaluations and Diagnostic/Therapeutic Applications of Chemically Designed Mesoporous Silica Nanoparticles," Advanced Materials 25, no. 23 (2013): 3144-3176, doi:10.1002/adma.201205292.

[11] Christian Argyo et al., "Multifunctional Mesoporous Silica Nanoparticles as a Universal Platform for Drug Delivery," Chemistry of Materials 26, no. 1 (January 14, 2014): 435-451, doi:10.1021/cm402592t.

[12] Ayush Verma and Francesco Stellacci, "Effect of Surface Properties on Nanoparticle-Cell Interactions," Small 6, no. 1 (2010): 12-21, doi:10.1002/smll.200901158.
[13] Andrea Kunzmann et al., "Toxicology of Engineered Nanomaterials: Focus on Biocompatibility, Biodistribution and Biodegradation," Biochimica et Biophysica Acta (BBA) - General Subjects 1810, no. 3 (March 2011): 361-373, doi:10.1016/j.bbagen.2010.04.007.

[14] Didem Sen Karaman et al., "Shape Engineering vs Organic Modification of Inorganic Nanoparticles as a Tool for Enhancing Cellular Internalization," Nanoscale Research Letters 7, no. 1 (December 1, 2012): 1-14, doi:10.1186/1556-276X-7-358.

[15] Hong Xu et al., "Preparation of Monodispersed Mesoporous Silica Spheres with Controllable Particle Size Under an Alkaline Condition," International Journal of Applied Ceramic Technology 9, no. 6 (2012): 1112-1123, doi:10.1111/j.17447402.2011.02716.x.

[16] Jessica M. Rosenholm et al., "Targeting of Porous Hybrid Silica Nanoparticles to Cancer Cells," ACS Nano 3, no. 1 (January 27, 2009): 197-206, doi:10.1021/nn800781r.

[17] Jessica M. Rosenholm, Antti Penninkangas, and Mika Lindén, "Amino-Functionalization of Large-Pore Mesoscopically Ordered Silica by a One-Step Hyperbranching Polymerization of a Surface-Grown Polyethyleneimine," Chemical Communications no. 37 (September 19, 2006): 3909-3911, doi:10.1039/B607886A.

[18] Jessica M. Rosenholm and Mika Lindén, "Wet-Chemical Analysis of Surface Concentration of Accessible Groups on Different Amino-Functionalized Mesoporous SBA-15 Silicas," Chemistry of Materials 19, no. 20 (October 1, 2007): 5023-5034, doi:10.1021/cm071289n.

[19] Jessica M. Rosenholm, Alain Duchanoy, and Mika Lindén, “Hyperbranching Surface Polymerization as a Tool for Preferential Functionalization of the Outer Surface of Mesoporous Silicat," Chemistry of Materials 20, no. 3 (February 1, 2008): 1126-1133, doi:10.1021/cm7021328.

[20] Rainer Wittig et al., "Active Targeting of Mesoporous Silica Drug Carriers Enhances $\Gamma$-Secretase Inhibitor Efficacy in an in Vivo Model for Breast Cancer," Nanomedicine (July 30, 2013): 1-17, doi:10.2217/nnm.13.62.

[21] Aurélien Auger et al., "A Comparative Study of Non-Covalent Encapsulation Methods for Organic Dyes into Silica Nanoparticles," Nanoscale Research Letters 6, no. 1 (April 13, 2011): 328, doi:10.1186/1556-276X-6-328.

[22] Daniel R. Larson et al., "Silica Nanoparticle Architecture Determines Radiative Properties of Encapsulated Fluorophores," Chemistry of Materials 20, no. 8 (April 1, 2008): 2677-2684, doi:10.1021/cm7026866.

[23] Robert Sjöback, Jan Nygren, and Mikael Kubista, "Absorption and Fluorescence Properties of Fluorescein," Spectrochimica Acta Part A: Molecular and Biomolecular Spectroscopy 51, no. 6 (June 1995): L7-L21, doi:10.1016/0584-8539(95)01421-P.

[24] A. Cook and A. Le, "The Effect of Solvent and pH on the Fluorescence Excitation and Emission Spectra of Solutions Containing Fluorescein.," J. Phy Chem Lab 10 (2006): 44-49.

[25] Jessica M. Rosenholm et al., "On the Nature of the Brønsted Acidic Groups on Native and Functionalized Mesoporous Siliceous SBA-15 as Studied by Benzylamine Adsorption from Solution," Langmuir 23, no. 8 (April 1, 2007): 4315-4323, doi:10.1021/la062450w.

[26] G.E.Ham, Polymeric Amines and Ammonium Salts, in: E.J. Goethals, ed., (Pergamon Press, Oxford, 1980). 
[27] O. Boussif et al., "A Versatile Vector for Gene and Oligonucleotide Transfer into Cells in Culture and in Vivo: Polyethylenimine," Proceedings of the National Academy of Sciences 92, no. 16 (August 1, 1995): 7297-7301.

[28] Yi-Ping Chen et al., "Surface Charge Effect in Intracellular Localization of Mesoporous Silica Nanoparticles as Probed by Fluorescent Ratiometric pH Imaging," RSC Advances 2, no. 3 (January 17, 2012): 968-973, doi:10.1039/C1RA00586C.

[29] Yanqing Tian et al., "Dually Fluorescent Sensing of pH and Dissolved Oxygen Using a Membrane Made from Polymerizable Sensing Monomers," Sensors and Actuators. B, Chemical 147, no. 2 (June 3, 2010): 714-722, doi:10.1016/j.snb.2010.03.029.

[30] W.T. Godbey, Kenneth K. Wu, and Antonios G. Mikos, "Poly(ethylenimine) and Its Role in Gene Delivery," Journal of Controlled Release 60, no. 2-3 (August 5, 1999): 149-160, doi:10.1016/S0168-3659(99)00090-5.

[31] Jixi Zhang, Diti Desai, and Jessica M. Rosenholm, "Tethered Lipid Bilayer Gates: Toward Extended Retention of Hydrophilic Cargo in Porous Nanocarriers," Advanced Functional Materials (2013): n/a-n/a, doi:10.1002/adfm.201302995.

[32] Werner Stöber, Arthur Fink, and Ernst Bohn, "Controlled Growth of Monodisperse Silica Spheres in the Micron Size Range," Journal of Colloid and Interface Science 26, no. 1 (January 1968): 6269, doi:10.1016/0021-9797(68)90272-5.

[33] Qianjun He et al., "The Three-Stage in Vitro Degradation Behavior of Mesoporous Silica in Simulated Body Fluid," Microporous and Mesoporous Materials 131, no. 1-3 (June 2010): 314-320, doi:10.1016/j.micromeso.2010.01.009.

[34] Siddharth V. Patwardhan, Graham E. Tilburey, and Carole C. Perry, "Interactions of Amines with Silicon Species in Undersaturated Solutions Leads to Dissolution And/or Precipitation of Silica," Langmuir 27, no. 24 (December 20, 2011): 15135-15145, doi:10.1021/la204180r.

[35] H. Takakusa et al., "Intramolecular Fluorescence Resonance Energy Transfer System with Coumarin Donor Included in BetaCyclodextrin," Analytical Chemistry 73, no. 5 (March 1, 2001): 939-942.

[36] Yasutomo Kawanishi et al., "Design and Synthesis of Intramolecular Resonance-Energy Transfer Probes for Use in Ratiometric Measurements in Aqueous Solution," Angewandte Chemie International Edition 39, no. 19 (2000): 34383440, doi:10.1002/1521-3773(20001002)39:19<3438::AIDANIE3438>3.0.CO;2-T.

[37] Ralph Iler, The Chemistry of Silica: Solubility, Polymerization, Colloid and Surface Properties and Biochemistry of Silica (New York: John Wiley and Sons, 1979).

[38] Jeffrey C. Brinker and George W. Scherer, Sol-Gel Science: The Physics and Chemistry of Sol-Gel Processing (Gulf Professional Publishing, 1990).

[39] Blake Simmons et al., "Understanding Amine Catalyzed Silica Polymerization: Diatoms as Bioarchitects” (Sandia National Laboratories, 2007).

[40] Rosa Casasús et al., "Toward the Development of Ionically Controlled Nanoscopic Molecular Gates," Journal of the American Chemical Society 126, no. 28 (July 1, 2004): 8612-8613, doi:10.1021/ja048095i.

[41] Rosa Casasús et al., "Dual Aperture Control on pH- and AnionDriven Supramolecular Nanoscopic Hybrid Gate-like Ensembles," Journal of the American Chemical Society 130, no. 6 (February 1, 2008): 1903-1917, doi:10.1021/ja0756772.
[42] C. Barbé et al., "Silica Particles: A Novel Drug-Delivery System," Advanced Materials 16, no. 21 (2004): 1959-1966, doi:10.1002/adma.200400771.

[43] Hong Dinh Duong and Jong II Rhee, "Exploitation of ThermoEffect of Rhodamine B Entrapped in Sol-gel Matrix and Silica Gel for Temperature Detection," Sensors and Actuators B: Chemical 124, no. 1 (June 10, 2007): 18-23, doi:10.1016/j.snb.2006.11.035.

[44] Igor Sokolov and Sajo Naik, "Novel Fluorescent Silica Nanoparticles: Towards Ultrabright Silica Nanoparticles," Small 4, no. 7 (2008): 934-939, doi:10.1002/smll.200700236.

[45] Eun-Bum Cho, Dmytro O. Volkov, and Igor Sokolov, "Ultrabright Fluorescent Mesoporous Silica Nanoparticles," Small 6, no. 20 (2010): 2314-2319, doi:10.1002/smll.201001337.

[46] Eun-Bum Cho, Dmytro O. Volkov, and Igor Sokolov, "Ultrabright Fluorescent Silica Mesoporous Silica Nanoparticles: Control of Particle Size and Dye Loading," Advanced Functional Materials 21, no. 16 (2011): 3129-3135, doi:10.1002/adfm.201100311.

[47] Didem Şen Karaman et al., "Rational Evaluation of the Utilization of PEG-PEI Copolymers for the Facilitation of Silica Nanoparticulate Systems in Biomedical Applications," Journal of Colloid and Interface Science 418 (March 15, 2014): 300-310, doi:10.1016/j.jcis.2013.11.080.

[48] Hervé Hillaireau and Patrick Couvreur, “Nanocarriers' Entry into the Cell: Relevance to Drug Delivery," Cellular and Molecular Life Sciences 66, no. 17 (September 1, 2009): 2873-2896, doi:10.1007/s00018-009-0053-z.

[49] Tore-Geir Iversen, Tore Skotland, and Kirsten Sandvig, "Endocytosis and Intracellular Transport of Nanoparticles: Present Knowledge and Need for Future Studies," Nano Today 6, no. 2 (April 2011): 176-185, doi:10.1016/j.nantod.2011.02.003.

[50] Joanna Rejman et al., "Size-Dependent Internalization of Particles via the Pathways of Clathrin- and Caveolae-Mediated Endocytosis.," Biochemical Journal 377, no. Pt 1 (January 1, 2004): 159-169, doi:10.1042/BJ20031253.

[51] Tsai-Hua Chung et al., "The Effect of Surface Charge on the Uptake and Biological Function of Mesoporous Silica Nanoparticles in 3T3-L1 Cells and Human Mesenchymal Stem Cells," Biomaterials 28, no. 19 (July 2007): 2959-2966, doi:10.1016/j.biomaterials.2007.03.006.

[52] Ilaria Rivolta, Panariti, and Miserocchi, "The Effect of Nanoparticle Uptake on Cellular Behavior: Disrupting or Enabling Functions?," Nanotechnology, Science and Applications (September 2012): 87, doi:10.2147/NSA.S25515.

[53] Nanjing Hao et al., "The Shape Effect of PEGylated Mesoporous Silica Nanoparticles on Cellular Uptake Pathway in Hela Cells," Microporous and Mesoporous Materials 162 (November 1, 2012): 14-23, doi:10.1016/j.micromeso.2012.05.040.

[54] Melissa D. Howard et al., "PEGylation of Nanocarrier Drug Delivery Systems: State of the Art," Journal of Biomedical Nanotechnology 4, no. 2 (June 1, 2008): 133-148, doi:10.1166/jbn.2008.021.

[55] Donald E. Owens III and Nicholas A. Peppas, "Opsonization, Biodistribution, and Pharmacokinetics of Polymeric Nanoparticles," International Journal of Pharmaceutics 307, no. 1 (January 3, 2006): 93-102, doi:10.1016/j.ijpharm.2005.10.010.

[56] Zohreh Amoozgar and Yoon Yeo, "Recent Advances in Stealth Coating of Nanoparticle Drug Delivery Systems," Wiley Interdisciplinary Reviews: Nanomedicine and Nanobiotechnology 4, no. 2 (2012): 219-233, doi:10.1002/wnan.1157. 
[57] Pablo del Pino et al., "Protein Corona Formation around Nanoparticles - from the Past to the Future," Materials Horizons (November 11, 2013), doi:10.1039/C3MH00106G.

[58] M. Laird Forrest, James T. Koerber, and Daniel W. Pack, "A Degradable Polyethylenimine Derivative with Low Toxicity for Highly Efficient Gene Delivery," Bioconjugate Chemistry 14, no. 5 (September 1, 2003): 934-940, doi:10.1021/bc034014g.

[59] He Shen et al., "Synthesis, Protein Delivery, and in Vitro and in Vivo Toxicity of a Biodegradable Poly(aminoester)," Toxicology Research 2, no. 6 (2013): 379, doi:10.1039/c3tx50074h.

[60] Veronika Mamaeva et al., "Mesoporous Silica Nanoparticles as Drug Delivery Systems for Targeted Inhibition of Notch Signaling in Cancer," Molecular Therapy 19, no. 8 (August 2011): 15381546, doi:10.1038/mt.2011.105.

[61] Hong Jin Kim, Joong Ho Moon, and Joon Won Park, "A Hyperbranched Poly(ethyleneimine) Grown on Surfaces," Journal of Colloid and Interface Science 227, no. 1 (July 1, 2000): 247-249, doi:10.1006/jcis.2000.6861.

[62] Tian Xia et al., "Polyethyleneimine Coating Enhances the Cellular Uptake of Mesoporous Silica Nanoparticles and Allows Safe Delivery of siRNA and DNA Constructs," ACS Nano 3, no. 10 (October 27, 2009): 3273-3286, doi:10.1021/nn900918w.

[63] Qianjun He et al., "Intracellular Localization and Cytotoxicity of Spherical Mesoporous Silica Nano- and Microparticles," Small 5, no. 23 (2009): 2722-2729, doi:10.1002/smll.200900923. 


\section{Supplementary information}

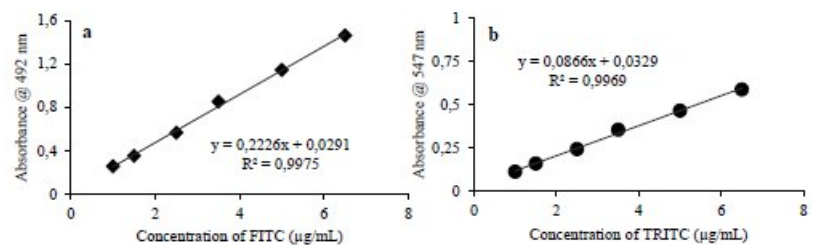

Fig. S1. Standard curves for a) FITC and b) TRITC fluorophores in $1 \mathrm{mg} / \mathrm{mL}$ dissolved MSNs in $2 \mathrm{M} \mathrm{NaOH}$ media. Supplementary

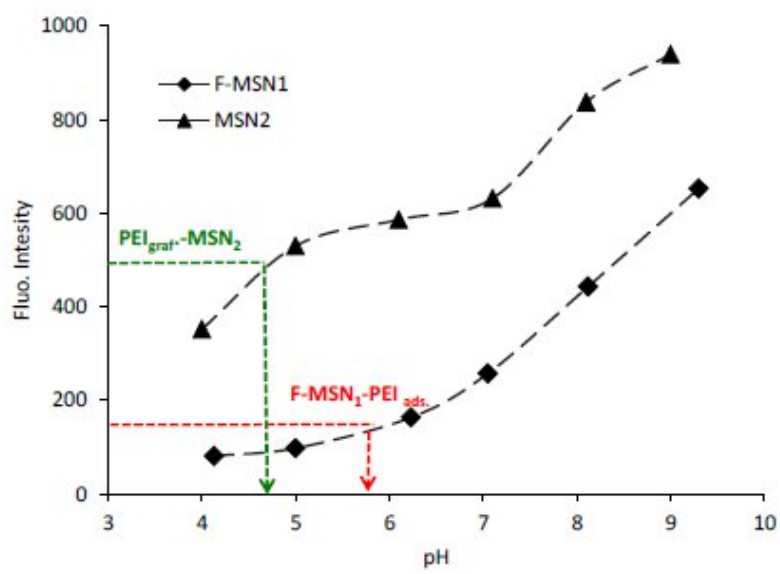

Fig. S2. pH-dependency of FITC-labelled MSNs used to probe the local pH experienced by fluorescein-molecules conjugated to their PEIfunctionalized counterparts.

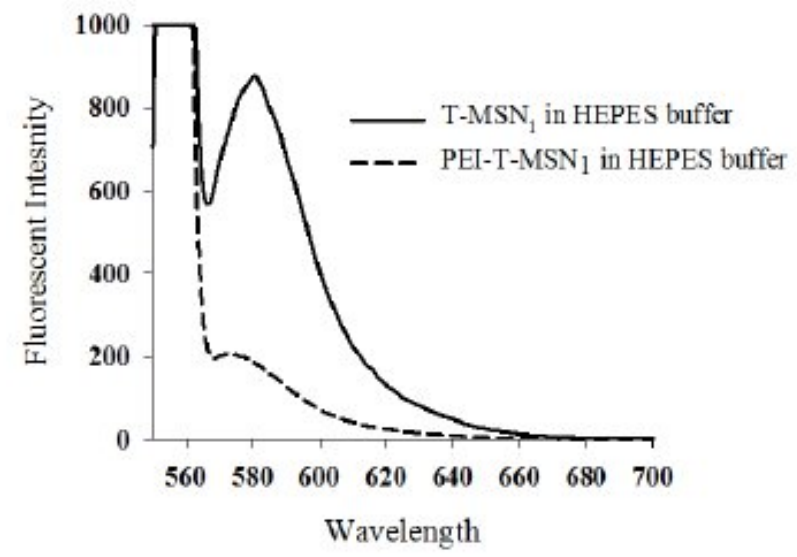

Fig. S3. Fluorescence intensity of rhodamine-labeled MSNs (T-MSNs) before and after PElcoating via electrostatic adsorption, using the same instrument settings. 


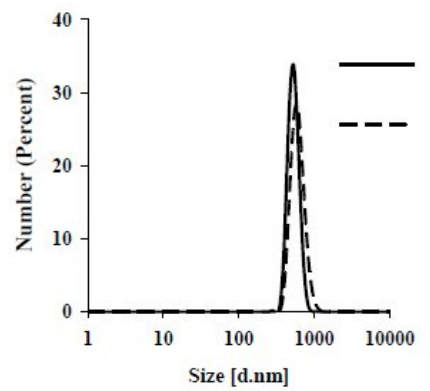

F-MSN, before leaching of dye F-MSN $\mathrm{N}_{1}$ after leaching of dye
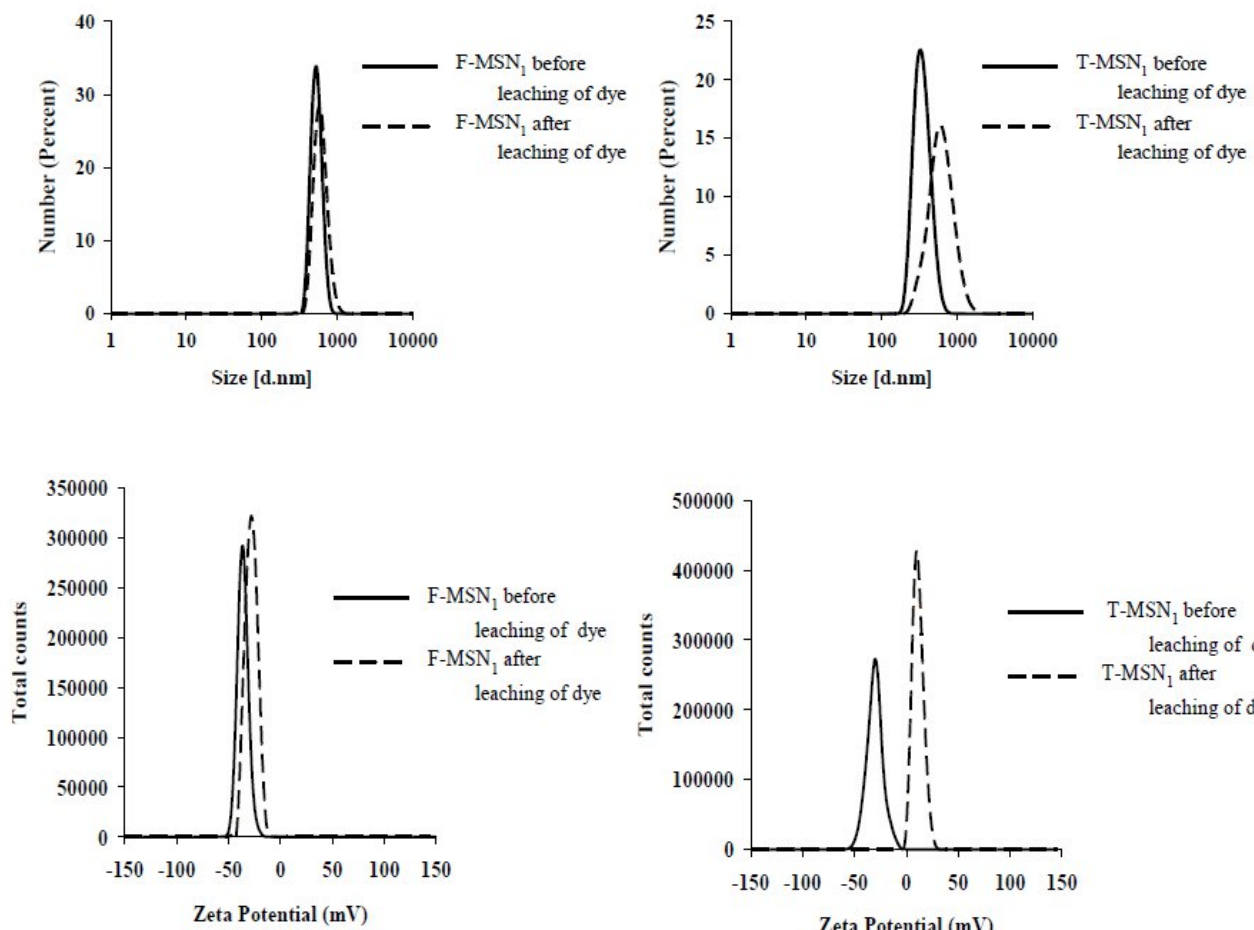

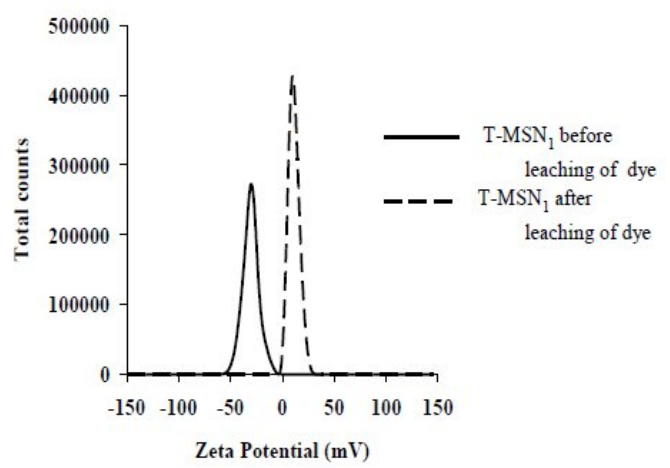

Fig. S4. Dynamic light scattering and zeta potential measurements of F-MSN1, PEI-F-MSN1, TMSN1 and PEI-T-MSN1s before and after fluorophore leaching studies (10 days incubation in HEPES at $37^{\circ} \mathrm{C}$ ).

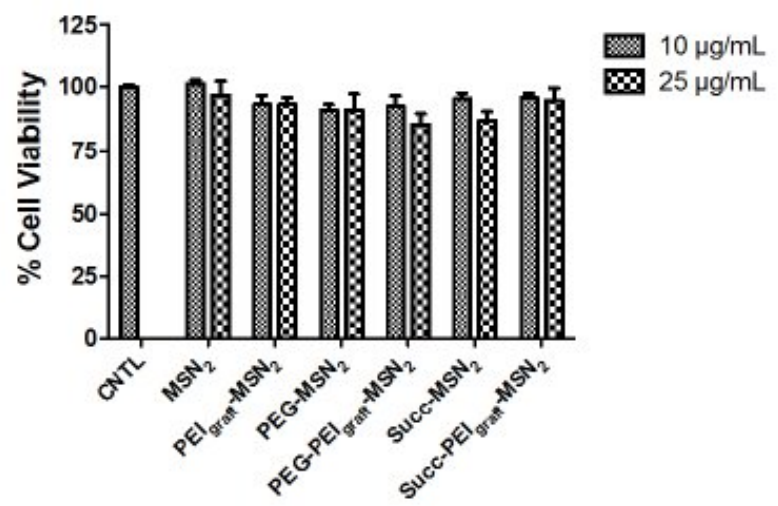

Type of Particle

Fig. S5. Cell viability of HeLa cells incubated with MSN2s for $72 \mathrm{~h}$ evaluated by the WST-1 assay. Data are expressed as mean \pm SEM of three to four independent experiments. 
a)

HeLa

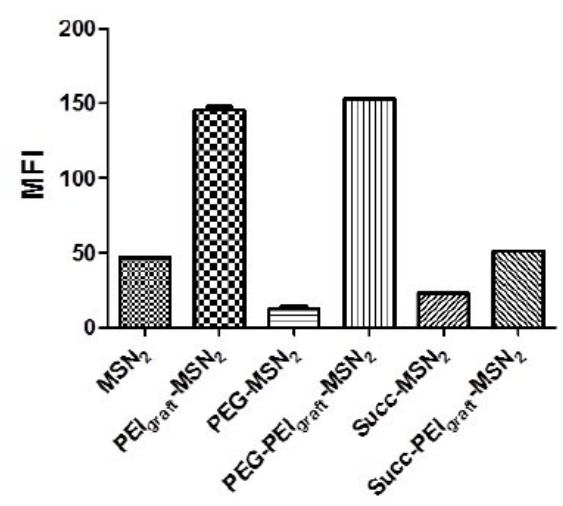

Type of Particle

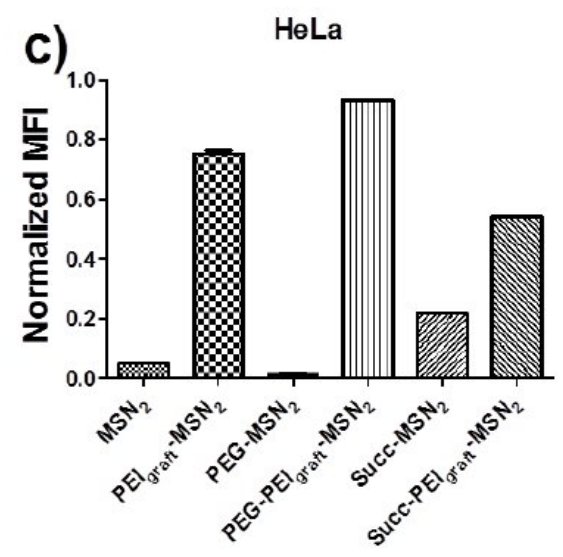

Type of Particle b)

Caco-2

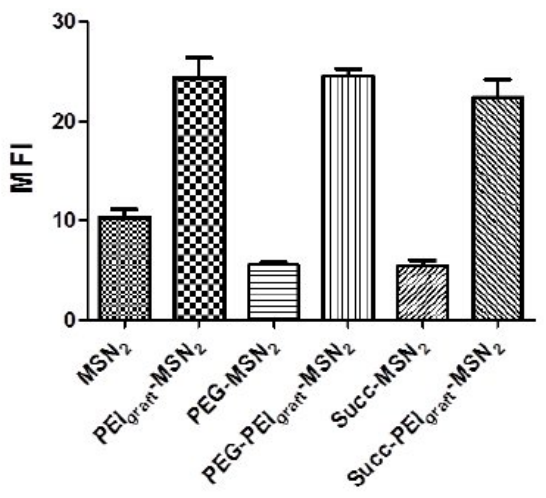

Type of Particle

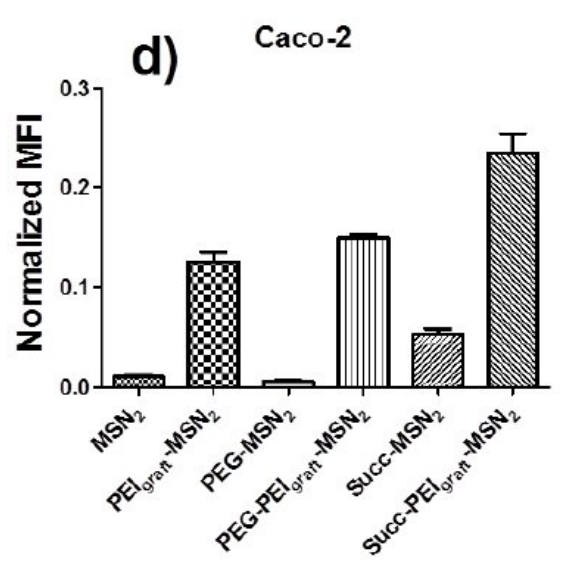

Type of Particle

Fig. S6. Flow cytometric investigation of MSNs $(5 \mu \mathrm{g} / \mathrm{mL}$ ) uptake after $4 \mathrm{~h}$ incubation in a) HeLa and b) Caco-2 cells, representing mean fluorescence intensities a-b) according to the raw data (highest, FL1-H) of MSNs, and c-d) as normalized against the fluorescence intensity value of specific MSN particle suspensions. 